\title{
On the analytic approximation of bulk collision rates of non-spherical hydrometeors
}

\author{
A. Seifert ${ }^{1}$, U. Blahak ${ }^{2}$, and R. Buhr ${ }^{3,4}$ \\ ${ }^{1}$ Hans-Ertel Centre for Weather Research, Deutscher Wetterdienst, Hamburg, Germany \\ ${ }^{2}$ Deutscher Wetterdienst, Offenbach, Germany \\ ${ }^{3}$ Universität Hamburg, Hamburg, Germany \\ ${ }^{4}$ Max Planck Institute for Meteorology, Hamburg, Germany \\ Correspondence to: A. Seifert (axel.seifert@dwd.de) \\ Received: 15 July 2013 - Published in Geosci. Model Dev. Discuss.: 25 September 2013 \\ Revised: 28 January 2014 - Accepted: 29 January 2014 - Published: 4 March 2014
}

\begin{abstract}
Analytic approximations of the binary collision rates of hydrometeors are derived for use in bulk microphysical parameterizations. Special attention is given to nonspherical hydrometeors like raindrops and snowflakes. The terminal fall velocity of these particles cannot be sufficiently well approximated by power-law relations which are used in most microphysical parameterizations, and therefore an improved formulation is needed. The analytic approximations of the bulk collision rates given in this paper are an alternative to look-up tables and can replace the Wisner approximation, which is used in many atmospheric models.
\end{abstract}

\section{Introduction}

The approximation of bulk collision rates is a classic problem in the formulation of cloud microphysical parameterizations for atmospheric models. The most common formulation is the continuous growth equation which applies to particles of very different sizes and fall speeds; i.e., the size and the fall speed of the smaller and thus slower falling particle is neglected (Rogers and Yau, 1996; Pruppacher and Klett, 1997; Straka, 2009). In practice, collisions of different particles of similar size and fall speed do occur, and one needs a formulation which is more general than the simple continuous growth equation. The standard approach for the collision rate of two ensembles of precipitation-sized particles goes back to Wisner et al. (1972), who used the ansatz that the velocity difference can be approximated by the difference of weighted means which then simplifies the solution of the collision integrals. If the weighting functions are properly chosen, this Wisner approximation can recover the continuous growth equations as asymptotic limits. The main disadvantage of this approach is that the bulk collision rate of the Wisner approximation becomes zero, if the difference of the weighted mean sedimentation velocities is zero, while for broad size distributions as they occur in nature the bulk collision rate is always non-zero.

Verlinde et al. (1990) studied analytic solution of the bulk collision integrals for generalized gamma distributions and terminal fall velocities which can be approximated by powerlaw relations. They provide exact solutions for this problem, but those include the general hypergeometric function and are therefore difficult and expensive to evaluate. Gaudet and Schmidt $(2005,2007)$ derived a generalized form of the Wisner approximation, which overcomes some of the deficiencies of the classic approximation. Straka and Gilmore (2006) discussed the effect of non-spherical raindrops on the bulk collision rate and argued that the usual approximation of spherical geometry in combination with a power-law fall speed is sufficiently accurate, but that this is the result of the cancellation of two relatively large errors. Ćurić and Janc (2010) showed that the use of size distributions without an upper cutoff diameter can lead to significant errors, especially for raindrops and hail.

An alternative to the Wisner approximation was suggested by Murakami (1990) and Mizuno (1990) and later by Seifert and Beheng (2006, SB2006 hereafter). The latter used the variance (or standard deviation) to approximate the bulk difference of the sedimentation velocities. Like most earlier 
parameterizations SB2006 limited their formulas to powerlaw relations for the terminal fall velocities.

In this paper we revisit the variance approximation introduced by SB2006. Especially for raindrops and snowflakes the power-law relations for the terminal fall velocity are not valid for large particles. Therefore new equations are derived which yield a better approximation for the collision rates that involve these particles. Additionally, the SB2006 variance ansatz is improved by introducing a more general weighting factor. For raindrops we include the non-spherical geometry of large raindrops for consistency with the more accurate terminal fall velocity approximation.

An alternative to analytic approximations of the integrals is look-up tables as they are used or recommended by many previous studies in their attempt to improve the parameterization of the collision integrals (e.g., Walko et al., 1995; Straka and Gilmore, 2006; Saleeby and Cotton, 2008; Thompson et al., 2008). In our opinion, look-up tables have some disadvantages and their use should be limited to cases where analytic solutions or approximations are not available. The reasons which made us discard the use of look-up tables are as follows:

1. Look-up tables increase the complexity of the modeling system because an automatic pre-processing becomes necessary which guarantees that the look-up tables are consistent with the microphysical parameters chosen for the specific simulation. This can become especially cumbersome in operational numerical weather prediction where reproducibility is of the essence.

2. Large multi-dimensional look-up tables can become inefficient because of the additional memory access, especially when the tables are larger than the cache size. On today's supercomputers atmospheric models are often memory bandwidth limited. Doing more floating point operations without additional memory access can thus be more efficient than using look-up tables.

3. Look-up tables are usually not included in the publications, nor are the corresponding source codes. This makes it difficult to reproduce the results from such models and hinders scientific progress.

4. While analytic approximations allow further theoretical studies, e.g., to explore the sensitivity to certain parameters or assumptions, this becomes limited to numerical studies once look-up tables have been introduced.

Therefore analytic approximations are in our opinion an important part of microphysical parameterizations. Another alternative to look-up tables are fits with, e.g., rational functions as used, for example, by Frick et al. (2013). In the end the question of which implementation strategy is most efficient depends very much on the application and the hardware architecture of the supercomputer and the processor.

The paper is organized as follows: in Sect. 2 we review and discuss the geometry and terminal fall velocity of raindrops and snowflakes and their approximation in bulk microphysical parameterizations. Sections 3 and 4 shortly introduce the Wisner and variance approximation of the bulk collision rates. In Sects. 5 and 6 we derive explicit parameterizations of the bulk collision rates of the collision between graupel and rain, and between snow and rain. Section 7 deals with the self-collection of snow. In Sect. 8 the previously introduced as well as other binary collision interactions are discussed by use of quantitative error measures. Some conclusions are given in Sect. 9. The Supplement contains auxiliary figures and further technical details on the collision interactions which are not in discussed in the main text.

\section{Geometry and fall speeds of raindrops and snowflakes}

Many hydrometeors in the atmosphere can be approximated as spheres, and this approximation is often made in bulk microphysical models. As is well known, the assumption of sphericity does not hold for raindrops larger than $1 \mathrm{~mm}$ diameter because the aerodynamic pressure forces lead to an oblate shape and oscillations (e.g., Beard and Chuang, 1987; Szakall et al., 2010). The axis ratio of raindrops

$\eta=\frac{D_{r, \min }}{D_{r, \max }}$,

where $D_{r, \min }$ and $D_{r, \max }$ are the minimum and maximum dimensions of the raindrop, is shown in Fig. 1a based on the theoretical model of Beard and Chuang (1987), the polynomial fit of Chuang and Beard (1990), and the parameterization following Khvorostyanov and Curry (2002) given by

$$
\begin{aligned}
\eta\left(D_{r, \max }\right) & =\exp \left(-\frac{D_{r, \text { max }}}{D_{\eta}}\right) \\
& +\left[1-\exp \left(-\frac{D_{r, \max }}{D_{\eta}}\right)\right]\left[\frac{1}{1+D_{r, \max } / D_{\eta}}\right] .
\end{aligned}
$$

The length scale $D_{\eta}$ is a constant which we chose to be $5.2 \mathrm{~mm}$. Compared to Khvorostyanov and Curry (2002) this is a slightly higher value, but it fits the data of Beard and Chuang (1987) better than the $4.7 \mathrm{~mm}$ used by Khvorostyanov and Curry (2002). Assuming a perfect oblate spheroid, the mass of the raindrop is given by $x_{r}=$ $(\pi / 6) \rho_{w} \eta D_{r, \max }^{3}$, i.e., given the equivalent diameter of the raindrop Eq. (2) constitutes an implicit equation for the maximum dimension.

For the parameterization of the bulk collision rate we need a simpler explicit equation for $D_{r, \max }$ as a function of the equivalent diameter $D_{r}$. In the following we use

$D_{r, \max }\left(D_{r}\right)=D_{r} \exp \left(\omega_{r} D_{r}\right)$ 
a) axis ratio of raindrops

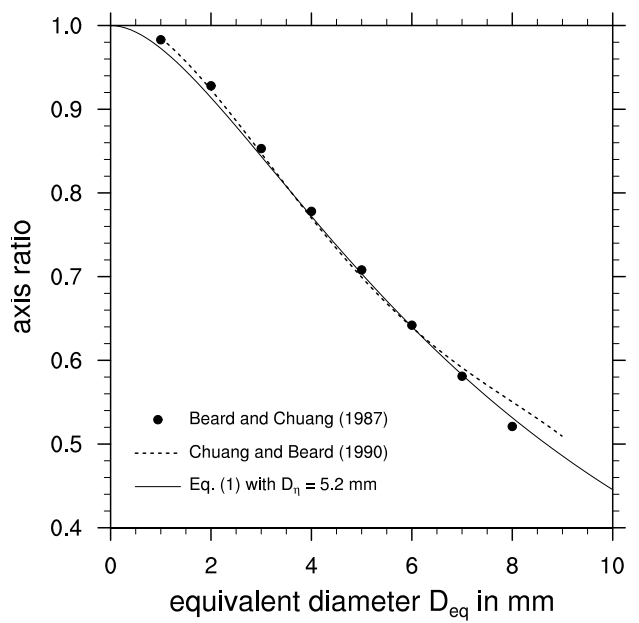

b) Maximum dimension of raindrops

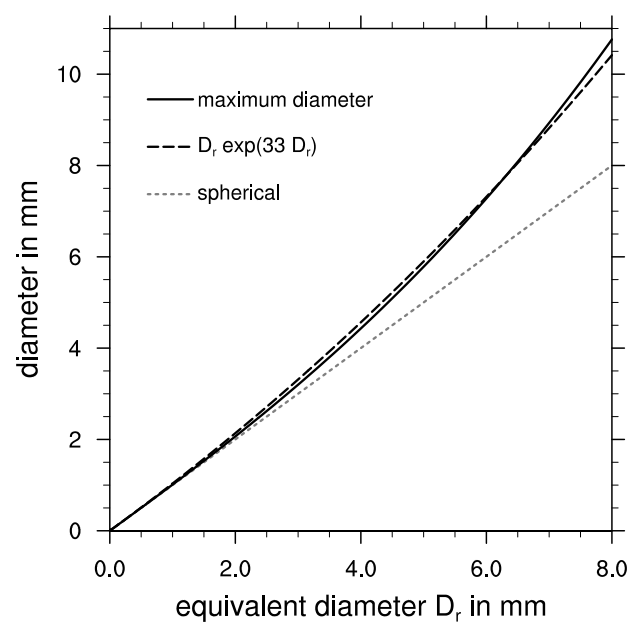

Fig. 1. Parameterization relations for non-spherical geometry of raindrops. The axis ratio as a function of equivalent diameter (left) following from Eq. (2) with $D_{\eta}=5.2 \mathrm{~mm}$, the results of the Beard and Chuang (1987) model and the polynomial fit of Chuang and Beard (1990). For the maximum diameter as a function of the equivalent diameter (right) the explicit relation $D_{r, \max }=D_{r} \exp \left(\omega_{r} D_{r}\right)$, with $\omega_{r}=33 \mathrm{~m}{ }^{-1}$, is used as an approximation to the implicit relation given by Eq. (2).

with $\omega_{r}=33 \mathrm{~m}^{-1}$, and Fig. 1b suggests that this is a sufficiently accurate approximation.

The fact that raindrops deviate from a spherical shape results in an increased cross-sectional area and a higher drag; thus it decreases the terminal fall velocity compared to a spherical particle of the same mass. Together with the high Reynolds number of a falling raindrop, i.e., the effects of turbulence, this explains the well-known empirical result that the terminal fall velocity of raindrops becomes independent of drop size for large drops, i.e., that the terminal fall velocity approaches a constant value. Many parameterizations of the terminal fall velocity of raindrops take this fact into account, e.g., the formulas by Best (1950), Atlas et al. (1973), Rogers et al. (1993) or Kogan and Shapiro (1996) give the correct asymptotics, i.e., a constant value at large diameters. Most of these relations are of the form

$v_{r}\left(D_{r}\right)=\alpha_{r}-\beta_{r} \exp \left(-\gamma_{r} D_{r}\right)$

or some variant thereof. Here $D_{r}$ is again the equivalent diameter of raindrops, and $\alpha_{r}, \beta_{r}$ and $\gamma_{r}$ are constant coefficients. In the following we will call Eq. (4) an Atlas-type fall speed relation. In contrast to that, bulk microphysical parameterizations traditionally use the less accurate power-law approximation for the terminal fall velocity (e.g., Kessler, 1969; Liu and Orville, 1969; Lin et al., 1983), which increases without bounds for large diameters.

Over the last decades an aerodynamic theory has been developed which predicts the terminal fall velocity of arbitrarily shaped particles over the whole size range, i.e, a large range of Reynolds numbers. The basic idea to apply boundary layer theory to the problem goes back to Abraham
(1970). Later Böhm (1989, 1992) showed that the boundary layer theory not only provides a powerful approach to treat the large range of Reynolds numbers but can also overcome the difficulties of arbitrarily shaped complicated particles. Böhm's approach was then further developed by Mitchell (1996) as well as Khvorostyanov and Curry (2002, 2005).

Figure 2 shows the terminal fall velocity of raindrops using the empirical relation of Beard (1976). The theoretical model of Khvorostyanov and Curry (2005, KC05 hereafter) agrees reasonably well with the empirical data, but underestimates the fall velocity of raindrops between 2 and $5 \mathrm{~mm}$ diameter. The simple power-law formula is as quite crude approximation and can only give a rough estimate over a limited size range. Especially for large raindrops the power law overestimates dramatically. In contrast, the Atlas-type fall speed relation provides a good approximation over a large size range. Here and in the following we have used the parameters

$\alpha_{r}=9.292 \mathrm{~ms}^{-1}, \beta_{r}=9.623 \mathrm{~ms}^{-1}, \gamma_{r}=6.222 \times 10^{2} \mathrm{~m}^{-1}$.

The only disadvantage is that they underestimate the terminal fall velocity of small drops; i.e., they do not approach the Stokes law and even become negative for very small drops. Nevertheless, for water drops larger than $0.1 \mathrm{~mm}$ diameter, i.e., for raindrops, it provides a very good parameterization.

Snowflakes show a similar behavior to raindrops in the sense that for large snowflakes the terminal fall velocity becomes approximately independent of size. The reason for this is that the particle bulk density decreases with increasing size of the snowflake; i.e., snowflakes grow faster than constant density spheres. The geometry of snowflakes can be described by a quadratic mass-size relation (Locatelli and 
a) terminal fall velocity of raindrops

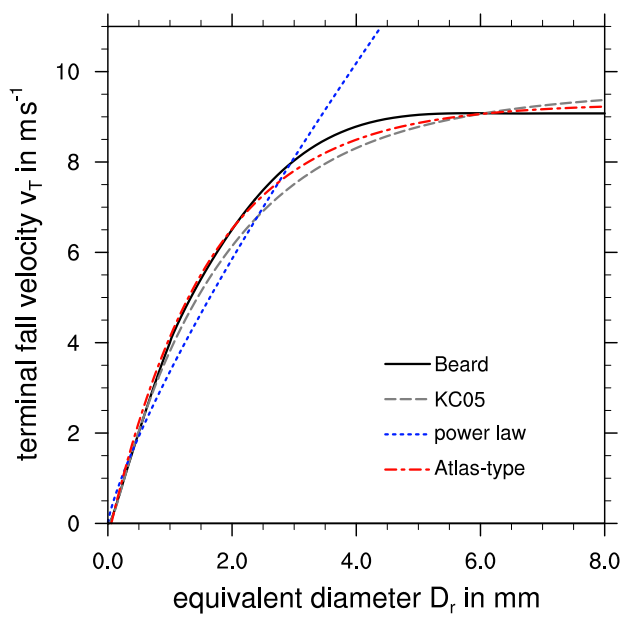

b) terminal fall velocity of raindrops (log-log axis)

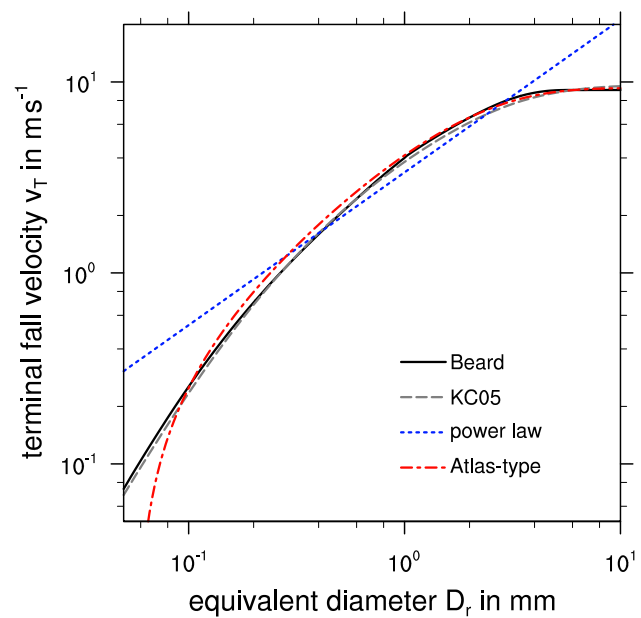

Fig. 2. Terminal fall velocity of raindrops as a function of equivalent diameter using different approaches. The empirical relation of Beard (black solid line) is regarded as the reference. The aerodynamic theory of Khvorostyanov and Curry (2002) can explain the empirical behavior, but significant differences exist (grey dashed line). The power-law approximation (blue dotted line) is in general inappropriate, but the Atlas-type relation (red dash-dotted line) gives a good approximation for raindrops larger than $0.1 \mathrm{~mm}$.

Hobbs, 1974; Brandes et al., 2007),

$x_{s}=a_{s} D_{s}^{2}$,

with the maximum dimension $D_{s}$, the mass of the snowflake $x_{s}$ and a constant parameter $a_{s}$. The quadratic mass-size relation is typical for growth regimes dominated by aggregation as shown by Westbrook et al. (2004a, b). Such relations are used in many bulk microphysical parameterizations; e.g., typical values for $a_{s}$ are between $0.038 \mathrm{~kg} \mathrm{~m}^{-2}$ (Doms and Schättler, 2002; Baldauf et al., 2011) and $a_{s}=0.069 \mathrm{~kg} \mathrm{~m}^{-2}$ (Wilson and Ballard, 1999; Field et al., 2005). Heymsfield (2003, his Eqs. 4 and 5) suggests that the lower value might be more appropriate for the tropics while the higher value is consistent with data from mid-latitudes.

Figure 3 shows the terminal fall velocity of low-density snowflakes with $a_{s}=0.038 \mathrm{~kg} \mathrm{~m}^{-2}$. To apply the KC05 aerodynamic model, an additional assumption about the cross-sectional area $A_{s}$ has to be made. Based on the observations of Field et al. (2008), namely the data presented in their Fig. 3, we chose for simplicity a constant area ratio $\hat{a}_{s}=A_{s} /\left[(\pi / 4) D_{s}^{2}\right]=0.45$. This should provide a good estimate for the small and medium-sized snowflakes, but might overestimate the cross-sectional area for snowflakes larger than $3 \mathrm{~mm}$ diameter and lead to an underestimation of the terminal fall velocity. Using, on the other hand, the area-size relation $A_{s}=0.2285 D_{s}^{1.88}$ of Mitchell (1996) for aggregates would result in about $20 \%$ lower terminal fall velocities than applying $\hat{a}_{s}=0.45$. The uncertainty in the terminal fall velocity of large snowflakes is obviously large, even for a given mass-size relationship.
For the Atlas-type approximation of resulting terminal fall velocity of snowflakes, we have the choice between a formulation using equivalent diameter

$v_{s}\left(D_{\text {eq }}\right)=\alpha_{s}-\beta_{s} \exp \left(-\gamma_{s} D_{\text {eq }}\right)$,

where $D_{\text {eq }}$ is the diameter of a liquid sphere, i.e.,

$D_{\text {eq }}=\left(\frac{6 x_{s}}{\pi \rho_{w}}\right)^{\frac{1}{3}}$,

and a formulation with maximum dimension

$v_{s}\left(D_{s}\right)=\tilde{\alpha}_{s}-\tilde{\beta}_{s} \exp \left(-\tilde{\gamma}_{s} D_{s}\right)$.

Note that we use $D_{\text {eq }}$ for the equivalent diameter of snow and $D_{r}$ for the equivalent diameter of raindrops. This is only an attempt to make some of the equations more readable, and both equivalent diameters are of course identical.

To optimize the parameters in these fits, we apply the Nelder-Mead downhill simplex method (Press et al., 1992), and with the resulting parameter values

$\alpha_{s}=1.271 \mathrm{~m} \mathrm{~s}^{-1}, \beta_{s}=1.252 \mathrm{~ms}^{-1}, \gamma_{s}=3.697 \times 10^{3} \mathrm{~m}^{-1}$ $\tilde{\alpha}_{s}=1.206 \mathrm{~m} \mathrm{~s}^{-1}, \tilde{\beta}_{s}=0.949 \mathrm{~ms}^{-1}, \tilde{\gamma}_{s}=1.654 \times 10^{3} \mathrm{~m}^{-1}$

we find that the formulation using the equivalent diameter $D_{\text {eq }}$ provides the more accurate fit to the KC05 terminal fall velocity. Especially from the log-log plot in Fig. 3b it becomes obvious that any power-law approximation can only provide a good fit for a small size range of snowflakes, but not for the whole relevant sizes range from, at least, $0.1 \mathrm{~mm}$ to $10 \mathrm{~mm}$ maximum dimension. 
For all hydrometeors used in this study, the geometries, terminal fall velocities and some further assumptions are summarized in Table 1. Hail is assumed as spherical particles with a (constant) particle density of $920 \mathrm{~kg} \mathrm{~m}^{-3}$. The cloud ice is hexagonal plates using the relations as given by Mitchell (1996), and for graupel we use a mass-size relation for lump graupel of Locatelli and Hobbs (1974). Note that these are not necessarily the particle properties assumed in the SB2006 two-moment scheme (or later publications using that scheme), but instead the particle properties here are chosen to span a wide, but typical, range of parameters. Some more details are discussed in Sects. 2 and 3 of the Supplement.

\section{Wisner approximation}

The classic gravitational collection kernel as it is found in most textbooks (e.g., Rogers and Yau, 1996; Pruppacher and Klett, 1997) is given by

$$
\begin{aligned}
K\left(D_{i}, D_{j}\right) & =\frac{\pi}{4}\left(D_{i}+D_{j}\right)^{2} \\
& \times\left|v_{i}\left(D_{i}\right)-v_{j}\left(D_{j}\right)\right| E_{i j}\left(D_{i}, D_{j}\right),
\end{aligned}
$$

with particle diameters $D_{i}, D_{j}$; terminal fall velocities $v_{i}, v_{j}$; and the collision-coalescence or aggregation efficiency $E_{i j}$. For oblate spheroids the relevant particle diameters are the maximum dimensions, while for more complicated geometries one would have to use an area-equivalent spherical diameter perpendicular to the fall direction. Assuming, without loss of generality, that $i$ is the collecting species and $j$ the one which is collected, the spectral collection rate for the species $j$, i.e., the loss term in the budget equation of the particle size distribution $f_{j}\left(D_{j}\right)$, is given by

$$
\begin{aligned}
\frac{\partial f_{j}\left(D_{j}\right)}{\partial t} & =-\frac{\pi}{4} \int_{0}^{\infty}\left(D_{i}+D_{j}\right)^{2} f_{i}\left(D_{i}\right) f_{j}\left(D_{j}\right) \\
& \times\left|v_{i}\left(D_{i}\right)-v_{j}\left(D_{j}\right)\right| E_{i j}\left(D_{i}, D_{j}\right) \mathrm{d} D_{i} .
\end{aligned}
$$

As usual, the particle size distribution $f(D)$ is defined as the number of particles per unit volume in the size range $[D, D+$ $\mathrm{d} D$ ]. Multiplication with $x_{j}^{n}$ and integration over the internal coordinate $D_{j}$ leads to the bulk collision rates for the integral moments of the collected species

$$
\begin{aligned}
\left.\frac{\partial M_{j, n}}{\partial t}\right|_{\text {coll }, i j} & =\frac{\pi}{4} \int_{0}^{\infty} \int_{0}^{\infty}\left(D_{i}+D_{j}\right)^{2} f_{i}\left(D_{i}\right) f_{j}\left(D_{j}\right) \\
& \times\left|v_{i}\left(D_{i}\right)-v_{j}\left(D_{j}\right)\right| E_{i j}\left(D_{i}, D_{j}\right) x_{j}^{n} \mathrm{~d} D_{i} \mathrm{~d} D_{j} ;
\end{aligned}
$$

i.e., $M_{j, 1}$ is the mass density $L_{j}$ of the collected species and $M_{j, 0}=N_{j}$ its number density. The corresponding tendencies of the collecting species are

$$
\frac{\partial N_{i}}{\partial t}=0 \quad \text { and } \quad \frac{\partial L_{i}}{\partial t}=-\frac{\partial L_{j}}{\partial t} .
$$

The integral in Eq. (11) is in general very hard to solve analytically because of the absolute value of the fall speed difference. As mentioned in the Introduction, Verlinde et al. (1990) derived a general analytic solution for particle distributions in the form of gamma distributions combined with power-law relations for the terminal fall velocities. However, this analytic solution leads to the general hypergeometric function, which by itself is very difficult to evaluate. In practice, the analytic solution is computationally as expensive as the numerical calculation of the integral itself. Therefore many schemes do still apply the approximation introduced by Wisner et al. (1972), who replaced the actual terminal fall velocity by some bulk mean value $\bar{v}$ which does not depend on $D$. Doing the same also for the collision efficiency yields

$$
\begin{aligned}
\left.\frac{\partial M_{j, n}}{\partial t}\right|_{\text {coll }, i j} & =\frac{\pi}{4} \bar{E}_{i j}\left|\bar{v}_{i, n}-\bar{v}_{j, n}\right| \int_{0}^{\infty} \int_{0}^{\infty}\left(D_{i}+D_{j}\right)^{2} \\
& \times f_{i}\left(D_{i}\right) f_{j}\left(D_{j}\right) x_{j}^{n} \mathrm{~d} D_{i} \mathrm{~d} D_{j},
\end{aligned}
$$

and with

$\mathcal{C}_{n, i j}=\int_{0}^{\infty} \int_{0}^{\infty}\left(D_{i}+D_{j}\right)^{2} f_{i}\left(D_{i}\right) f_{j}\left(D_{j}\right) x_{j}^{n} \mathrm{~d} D_{i} \mathrm{~d} D_{j}$

we can write this as

$$
\left.\frac{\partial M_{j, n}}{\partial t}\right|_{\mathrm{coll}, i j}=\frac{\pi}{4} \bar{E}_{i j}\left|\bar{v}_{i, n}-\bar{v}_{j, n}\right| \mathcal{C}_{n, i j} .
$$

With the usual assumptions about the particle size distributions, the remaining integral in $\mathcal{C}_{n, i j}$ can be solved quite easily.

Wisner et al. (1972) specified both bulk terminal fall velocities as the mass-weighted fall speeds. A more detailed analysis of the asymptotic behavior, which should recover the continuous growth equations, shows that the bulk terminal fall velocity of the collecting particles should be weighted with $D^{2}$ while the bulk fall speed of the collected particles has to be weighted with $D^{2} x$ (Seifert, 2002). When using a two-moment scheme the equation for the number densities should apply $D^{2}$-weighted fall speeds for both species. The bulk velocities in the Wisner approximation are therefore calculated by

$\bar{v}_{i, n}=\frac{\int_{0}^{\infty} v_{i}\left(D_{i}\right) D_{i}^{2} f_{i}\left(D_{i}\right) \mathrm{d} D_{i}}{\int_{0}^{\infty} D_{i}^{2} f_{i}\left(D_{i}\right) d D_{i}}$

$\bar{v}_{j, n}=\frac{\int_{0}^{\infty} v_{j}\left(D_{j}\right) D_{j}^{2} f_{j}\left(D_{j}\right) x_{j}^{n} \mathrm{~d} D_{j}}{\int_{0}^{\infty} D_{j}^{2} f_{j}\left(D_{j}\right) x_{j}^{n} \mathrm{~d} D_{j}}$,

and $n=0$ applies to number density, and $n=1$ to the mass density equation. Note that for the collecting species the bulk velocity does not depend on $n$; i.e., the same velocity is used for all moments. 
a) terminal fall velocity of snowflakes

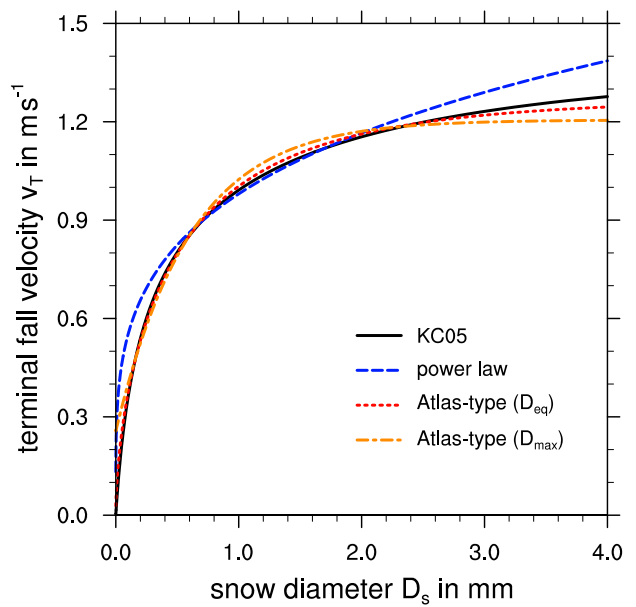

b) terminal fall velocity of snowflakes (lin-log axis)

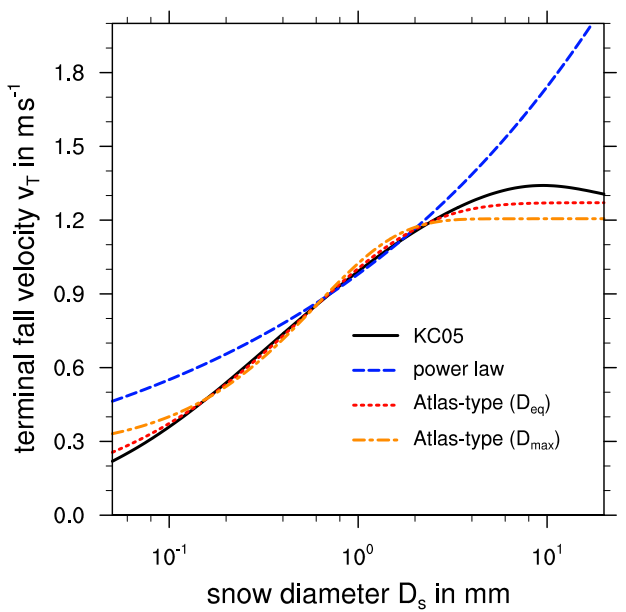

Fig. 3. Terminal fall velocity of snowflakes with the mass-size relation $x=0.038 D_{s}^{2}$ and a constant area ratio of $\hat{a}_{s}=0.45$. As a reference we use the aerodynamic theory of Khvorostyanov and Curry $(2002,2005)$ (black solid line). The approximations are a power-law (blue dashed) and an Atlas-type relation using either equivalent diameter (red dashed) or the maximum dimension (orange dashed).

Table 1. Coefficients for the mass-size relation $x=\hat{a} D^{\hat{b}}$, the maximum dimension as a function of particle mass $D=a x^{b}$, the power-law terminal fall velocity $v(x)=\alpha x^{\beta}$ of particles with mass $x$ and the shape parameters of the gamma distributions $f(x)=A x^{\nu} \exp \left(-B x^{\xi}\right)$ and $f\left(D_{\mathrm{eq}}\right)=N_{0} D_{\mathrm{eq}}^{\mu} \exp \left(-\lambda D_{\mathrm{eq}}\right)$. Note that for the raindrops only the spherical geometry is given here, but the non-spherical correction is taken into account explicitly by $D_{r, \max }=D_{r} \exp \left(\omega_{r} D_{r}\right)$. For the area-size relation we give the pre-factor $\gamma_{A}$ in the formula $A=\gamma_{A} D^{2}$, where $A$ is the cross-sectional area and $D$ is the maximum dimension.

\begin{tabular}{lrrrrrrrrrr}
\hline & $\hat{a}$ & $\hat{b}$ & $a$ & $b$ & $\alpha$ & $\beta$ & $v$ & $\xi$ & $\mu$ & $\gamma_{A}$ \\
\hline raindrops & $\pi \rho_{w} / 6$ & 3.0 & - & - & 159.0 & 0.2667 & 0.0 & $1 / 3$ & 2.0 & $\pi / 4$ \\
snowflakes & 0.038 & 2.0 & 5.13 & 0.5 & 8.294 & 0.125 & 0.0 & $1 / 3$ & 2.0 & $0.45(\pi / 4)$ \\
graupel & 19.51 & 2.8 & 0.346 & 0.357 & 17.5 & 0.17 & 1.0 & 1.0 & - & $\pi / 4$ \\
hail & 500.1 & 3.18 & 0.142 & 0.314 & 33. & 0.187 & 1.0 & $1 / 3$ & 5.0 & $\pi / 4$ \\
cloud ice & 1.588 & 2.564 & 0.835 & 0.390 & 27.7 & 0.216 & 0.0 & $1 / 3$ & 2.0 & $3 / 8 \sqrt{3}$ \\
\hline
\end{tabular}

\section{Variance approximation}

The Wisner approximation has one major disadvantage: the collision rate becomes zero when the two bulk velocities are equal. For polydisperse particle size distributions this is, of course, not consistent with the original collection equation. The true bulk collection rate will have a minimum somewhere, but it never becomes zero. One could argue that the small collision rates close to that minimum can be neglected anyway, but this may be a false conclusion. For example, when graupel grows by collection of raindrops, then the small graupel particles have fall speeds which are smaller than those of raindrops, while the large graupel may have higher fall speeds than rain; i.e., during this growth the graupel has to go through the minimum in the collision rate. If the bulk collision rate becomes zero due to the Wisner approximation, it might significantly slow down the growth of graupel because just by collecting rain it is impossible to overcome this singularity.
Murakami (1990) and Mizuno (1990) suggested a new formulation of the Wisner approximation in which they replaced the difference of the weighted means in Eq. (15) by an ad hoc parameterization of the velocity difference.

$$
\begin{aligned}
\left.\frac{\partial M_{j, n}}{\partial t}\right|_{\text {coll }, i j} & =\frac{\pi}{4} \bar{E}_{i j} \mathcal{C}_{n, i j} \\
& \times \sqrt{\left(\alpha_{M} \bar{v}_{i, n}-\beta_{M} \bar{v}_{j, n}\right)^{2}+\gamma_{M} \bar{v}_{i, n} \bar{v}_{j, n}},
\end{aligned}
$$

with $\alpha_{M 90}=\beta_{M 90}=1$ and $\gamma_{M 90}=0.04$ as given by Murakami (1990), while Mizuno (1990) uses different values. This parameterization is, for example, used by Mansell et al. (2010) as well as Milbrandt and Yau (2005b). The disadvantage of this ad hoc formulation is that the necessary coefficients cannot be derived, and it is questionable whether one set of coefficients is good for all possible particle types and combinations.

Inspired by the parameterization of Murakami and Mizuno, SB2006 introduced an approximation in which they 
parameterize the bulk velocity difference by the square root of the second moment of the velocity differences, a quantity which can be calculated analytically.

For the SB2006 variance formulation we write the Wisner approximation as

$$
\left.\frac{\partial M_{j, n}}{\partial t}\right|_{\mathrm{coll}, i j}=\frac{\pi}{4} \bar{E}_{i j} \overline{\Delta v}_{n, i j} \mathcal{C}_{n, i j}
$$

and the bulk velocity difference is parameterized as

$$
\begin{aligned}
\overline{\Delta v}_{n, i j} & =\left\{\frac{1}{\mathcal{N}_{n, i j}} \int_{0}^{\infty} \int_{0}^{\infty}\left[v_{i}\left(D_{i}\right)-v_{j}\left(D_{j}\right)\right]^{2}\right. \\
& \left.\times D_{i}^{2} D_{j}^{2} f_{i}^{m}\left(D_{i}\right) f_{j}^{m}\left(D_{j}\right) x_{j}^{n} d D_{i} d D_{j}\right\}^{\frac{1}{2}},
\end{aligned}
$$

with the normalization factor $\mathcal{N}_{n, i j}$ given by

$\mathcal{N}_{n, i j}=\int_{0}^{\infty} \int_{0}^{\infty} D_{i}^{2} D_{j}^{2} f_{i}^{m}\left(D_{i}\right) f_{j}^{m}\left(D_{j}\right) x_{j}^{n} d D_{i} d D_{j}$.

Here we have introduced an additional exponent $m$ following a suggestion of Blahak (2012). SB2006 originally proposed $m=1$, but this choice is not the only possible one. For the exponent $m$ values between one and two seem reasonable. This exponent modifies the weight of both size distributions, and, e.g, by giving more weight to higher moments it essentially shifts the minimum of the collision rate along the internal coordinate (see Sect. 5 and Fig. 16 of the Supplement for further details).

\section{Graupel-rain collection rates}

To specify the integrals of the previous section for the riming rate of graupel collecting rain, we make the following assumptions

$$
\begin{aligned}
f_{r}\left(D_{r}\right) & =N_{0, r} D_{r}^{\mu_{r}} \exp \left(-\lambda_{r} D_{r}\right) \\
f_{g}\left(x_{g}\right) & =A_{g} x^{v_{g}} \exp \left(-B_{g} x_{g}^{\xi_{g}}\right) \\
D_{g} & =a_{g} x_{g}^{b_{g}} \\
x_{r} & =\frac{\pi}{6} \rho_{w} D_{r}^{3} \\
v_{g} & =\alpha_{g} x_{g}^{\beta_{g}} \\
v_{r} & =\alpha_{r}-\beta_{r} \exp \left(-\gamma_{r} D_{r}\right) \\
D_{r, \max } & =D_{r} \exp \left(\omega_{r} D_{r}\right) .
\end{aligned}
$$

First, we solve for the integral including the cross-sectional area

$$
\begin{aligned}
\mathcal{C}_{n, g r} & =\int_{0}^{\infty} \int_{0}^{\infty}\left[D_{g}+D_{r, \max }\right]^{2} \\
& \times f_{r}\left(D_{r}\right) f_{g}\left(D_{g}\right) x_{r}^{n} \mathrm{~d} D_{r} \mathrm{~d} D_{g} .
\end{aligned}
$$

With the bulk quantities defined as $\bar{x}_{j}=L_{j} / N_{j}$ and $\bar{D}_{j}=$ $D_{j}\left(\bar{x}_{j}\right)=a_{j} \bar{x}_{j}^{b_{j}}$ for any species $j \in\{r, g, i, s\}$ we find

$$
\begin{aligned}
\mathcal{C}_{n, g r} & =N_{g} N_{r} \bar{x}_{r}^{n}\left[\delta_{g, 2}^{*} \bar{D}_{g}^{2}+2 \delta_{g, 1}^{*} \bar{D}_{g}\right. \\
& \times \frac{\lambda_{r}^{\mu+3 n+1}}{\left(\lambda_{r}-\omega_{r}\right)^{\mu_{r}+3 n+2}} \frac{\Gamma\left(\mu_{r}+3 n+2\right)}{\Gamma^{1-n}\left(\mu_{r}+1\right) \Gamma^{n}\left(\mu_{r}+4\right)} \\
& \left.+\frac{\lambda_{r}^{\mu_{r}+3 n+1}}{\left(\lambda_{r}-2 \omega_{r}\right)^{\mu_{r}+3 n+3}} \frac{\Gamma\left(\mu_{r}+3 n+3\right)}{\Gamma^{1-n}\left(\mu_{r}+1\right) \Gamma^{n}\left(\mu_{r}+4\right)}\right]
\end{aligned}
$$

and

$\delta_{g, k}^{*}=\frac{\Gamma\left(\frac{k b_{g}+v_{g}+1}{\xi_{g}}\right)}{\Gamma\left(\frac{\nu_{g}+1}{\xi_{g}}\right)}\left[\frac{\Gamma\left(\frac{v_{g}+1}{\xi_{g}}\right)}{\Gamma\left(\frac{v_{g}+2}{\xi_{g}}\right)}\right]^{k b_{g}}$,

where $\delta_{g, 2}^{*}$ is equal to $\delta_{g}^{0}$ of Eq. (90) of SB2006. Here $\Gamma(\xi)$ is the gamma function which we evaluate using the method given in Press et al. (1992). With Fortran 2008, $\Gamma(\xi)$ becomes part of the Fortran standard and optimized codes should become readily available. Nevertheless, the evaluation of the gamma function remains time consuming in these relations. In most two-moment bulk schemes the shape parameters like $\mu_{r}, v_{g}$, or $\xi_{g}$ and the particle properties that determine, e.g., $b_{g}$ or $\beta_{g}$ are constant during a simulation, and therefore parameters like $\delta_{g, k}^{*}$ or $\vartheta_{p, m, k}^{*}$, as given by Eq. (33) below, have to be calculated only once at the initialization of the microphysics scheme. However, the behavior of the particle sedimentation can be improved by using a diagnostic $\mu-\lambda$ or shape-slope relation, i.e., by relating the shape parameters of the size distributions to the slope or mean size of the distribution (Milbrandt and Yau, 2005a; Seifert, 2008; Milbrandt and McTaggart-Cowan, 2010). Unfortunately, this makes it then necessary to recalculate the coefficients which include the gamma function during runtime. A compromise is to use the shape-slope relation only for precipitation-sized particles outside the cloud where the gravitational sorting is dominant, but to revert to a constant shape parameter inside the clouds where the size distribution is dominated by collisioncoalescence and other growth processes. By doing so, most parameters become again constant coefficients, and only a few change with time. Note that with the new parameterizations suggested here, fewer gamma functions occur in the relations compared to the SB2006 parameterization, which is based purely on power laws.

For the bulk velocity difference the assumptions lead to

$$
\begin{aligned}
{\overline{\Delta v_{n, g r}}}_{2} & =\vartheta_{g, m, 2}^{*} \bar{v}_{g}^{2}-\vartheta_{g, m, 1}^{*} \bar{v}_{g}\left[\alpha_{r}-\beta_{r}\left(1+\frac{\gamma_{r}}{m \lambda_{r}}\right)^{\zeta}\right] \\
& +\alpha_{r}^{2}-2 \alpha_{r} \beta_{r}\left(1+\frac{\gamma_{r}}{m \lambda_{r}}\right)^{\zeta}+\beta_{r}^{2}\left(1+\frac{2 \gamma_{r}}{m \lambda_{r}}\right)^{\zeta},
\end{aligned}
$$


with $\zeta=3 n+m \mu_{r}+1$ and $\bar{v}_{j}=v_{j}\left(\bar{x}_{j}\right)$ and

$\vartheta_{p, m, k}^{*}=\frac{\Gamma\left(\frac{k \beta_{p}+2 b_{p}+m v_{p}+k+1}{\xi_{p}}\right)}{\Gamma\left(\frac{2 b_{p}+m v_{p}+k+1}{\xi_{p}}\right)}\left[\frac{\Gamma\left(\frac{v_{p}+1}{\xi_{p}}\right)}{m \Gamma\left(\frac{v_{p}+2}{\xi_{p}}\right)}\right]^{\beta_{p}}$,

which is a coefficient similar, but not equal, to Eq. (92) of SB2006. Using these approximations the rate equations for all moments can be parameterized. For number and mass densities of rain we find

$$
\begin{aligned}
& \left.\frac{\partial N_{r}}{\partial t}\right|_{\text {coll, }, g r}=-\frac{\pi}{4} N_{g} N_{r} \bar{E}_{g r} \\
& \times\left[\delta_{g, 2}^{*} \bar{D}_{g}^{2}+2 \delta_{g, 1}^{*} \bar{D}_{g}(\mu+1) \frac{\lambda_{r}^{\mu+1}}{\left(\lambda_{r}-\omega_{r}\right)^{\mu+2}}\right. \\
& \left.+(\mu+1)(\mu+2) \frac{\lambda_{r}^{\mu+1}}{\left(\lambda_{r}-2 \omega_{r}\right)^{\mu+3}}\right] \\
& \quad \times\left[\vartheta_{g, m, 2}^{*} \bar{v}_{g}^{2}-\vartheta_{g, m, 1}^{*} \bar{v}_{g}\left(\alpha_{r}-\beta_{r}\left(1+\frac{\gamma_{r}}{m \lambda_{r}}\right)^{m \mu_{r}+1}\right)\right. \\
& \left.\quad+\alpha_{r}^{2}-2 \alpha_{r} \beta_{r}\left(1+\frac{\gamma_{r}}{m \lambda_{r}}\right)^{m \mu_{r}+1}+\beta_{r}^{2}\left(1+\frac{2 \gamma_{r}}{m \lambda_{r}}\right)^{m \mu_{r}+1}\right]^{\frac{1}{2}}
\end{aligned}
$$

and

$$
\begin{aligned}
& \left.\frac{\partial L_{r}}{\partial t}\right|_{\text {coll, }, r}=-\frac{\pi}{4} N_{g} L_{r} \bar{E}_{g r} \\
& \times\left[\delta_{g, 2}^{*} \bar{D}_{g}^{2}+2 \delta_{g, 1}^{*} \bar{D}_{g}(\mu+4) \frac{\lambda_{r}^{\mu+4}}{\left(\lambda_{r}-\omega_{r}\right)^{\mu+5}}\right. \\
& \left.+(\mu+4)(\mu+5) \frac{\lambda_{r}^{\mu+4}}{\left(\lambda_{r}-2 \omega_{r}\right)^{\mu+6}}\right] \\
& \times\left[\vartheta_{g, m, 2}^{*} \bar{v}_{g}^{2}-\vartheta_{g, m, 1}^{*} \bar{v}_{g}\left(\alpha_{r}-\beta_{r}\left(1+\frac{\gamma_{r}}{m \lambda_{r}}\right)^{m \mu_{r}+4}\right)\right. \\
& \left.+\alpha_{r}^{2}-2 \alpha_{r} \beta_{r}\left(1+\frac{\gamma_{r}}{m \lambda_{r}}\right)^{m \mu_{r}+4}+\beta_{r}^{2}\left(1+\frac{2 \gamma_{r}}{m \lambda_{r}}\right)^{m \mu_{r}+4}\right]^{\frac{1}{2}} .
\end{aligned}
$$

The resulting approximations using these equations in comparison with the Wisner approximation and the equations given by SB2006 are shown in Figs. 4 and 5, in which the normalized bulk collision rates

$$
\begin{aligned}
\mathcal{K}_{N, g r} & =\left.\frac{1}{N_{r} N_{g}\left(\bar{D}_{r}+\bar{D}_{g}\right)^{2}} \frac{\partial N_{r}}{\partial t}\right|_{\text {coll }, g r} \\
\mathcal{K}_{L, g r} & =\left.\frac{1}{L_{r} N_{g}\left(\bar{D}_{r}+\bar{D}_{g}\right)^{2}} \frac{\partial L_{r}}{\partial t}\right|_{\text {coll }, g r}
\end{aligned}
$$

are compared with the numerical solution. Note that the normalized bulk collision rates have units of $\mathrm{m} \mathrm{s}^{-1}$; i.e., they can also be interpreted as collection velocities. These normalized rates or characteristic bulk collection velocities of number and mass give a better visual impression of the agreement with the numerical solution than the collision rates themselves, because the trivial dependencies on the number or mass density and the cross-sectional area have been removed. For the numerical solution we have applied the Berry and Reinhardt (1973) higher-order finite-difference method on a logarithmic grid with 450 bins (mass doubling every 8 th bin). For the particle size distribution of graupel a generalized gamma distribution is assumed with a graupel mass density $L_{g}=1 \mathrm{~g} \mathrm{~m}^{-3}$ and shape parameters $v_{g}=1$ and $\xi_{g}=1$. For rain we assume a gamma distribution in equivalent diameter with $\mu_{r}=2$ and $L_{r}=1 \mathrm{~g} \mathrm{~m}^{-3}$.

The Figs. 4a and 5a show the well-known problems of the Wisner approximation, namely that the collision rate becomes zero where the correct solution has its local minimum. This is rectified by the variance approximation, and visually the SB2006 formulation is superior to the Wisner approximation using power-law fall speeds. Using the more accurate Atlas-type approximation of the terminal fall velocity of raindrops results in a much better agreement with the numerical solution, especially for large mean raindrop diameters. This is the case for both the Murakami and Mizuno (Figs. 4c and 5c) as well as the variance approximation (Figs. 4d and 5d). Note that this improvement is only achieved by taking into account both the Atlas-type fall speed and the non-spherical geometry of the raindrops, while the approximation that apply power-law fall speeds gives better results when combined with a purely spherical geometry as already pointed out by Straka and Gilmore (2006). This error compensation is probably not coincidence, but reflects the consistency of the assumptions. The agreement of the new variance formulation with the numerical solution is very good for the mass collision rate, but larger errors do occur for the number rate, as is also the case for the other approximations. For both collision rates the optimal values for the tuning parameter, $m=1.6$ for the mass rate and $m=2$ for the number rate, have been used for the new variance approximation (cf. Figs. 13 and 14 of the Supplement and corresponding text). The parameterization of Murakami and Mizuno gives a result for the mass rate which is almost as good as for our new scheme, but is slighly worse for the number rate. Note that we did not retune and optimize the parameters of the Murakami-Mizuno ansatz, but simply applied the values of Murakami (1990). A quantitative error analysis is discussed in Sect. 8.

\section{Snow-rain collection rates}

The collection rate of snow and rain is an example of the case of both terminal fall velocities being approximated by Atlas-type relations. For the raindrops we do again take into account the non-spherical correction, but for the snowflakes this is already included in the $x_{s} \sim D_{s}^{2}$ relationship. Combined with two gamma distributions in equivalent diameter the assumptions are

$$
f_{r}\left(D_{r}\right)=N_{0, r} D_{r}^{\mu_{r}} \exp \left(-\lambda_{r} D_{r}\right)
$$


a) Wisner approx. using power-law fall speed

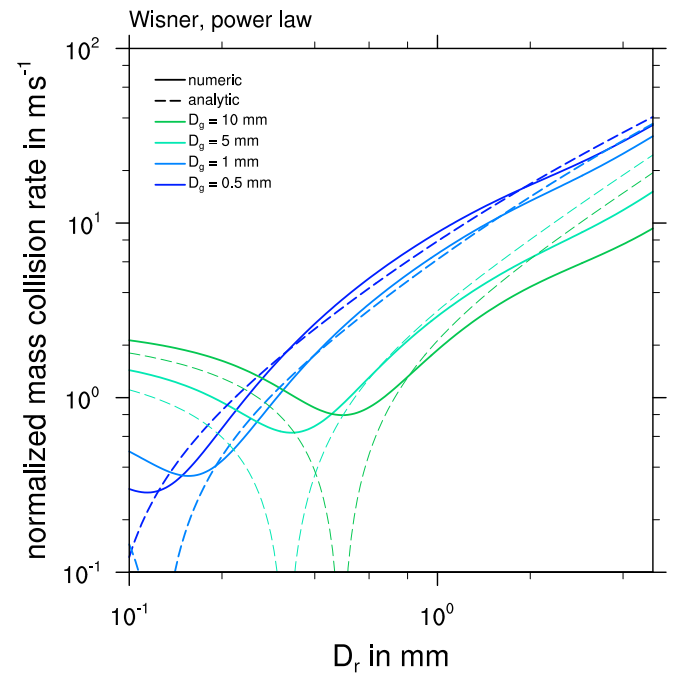

c) Murakami-Mizuno approx. using Atlas-type fall speed

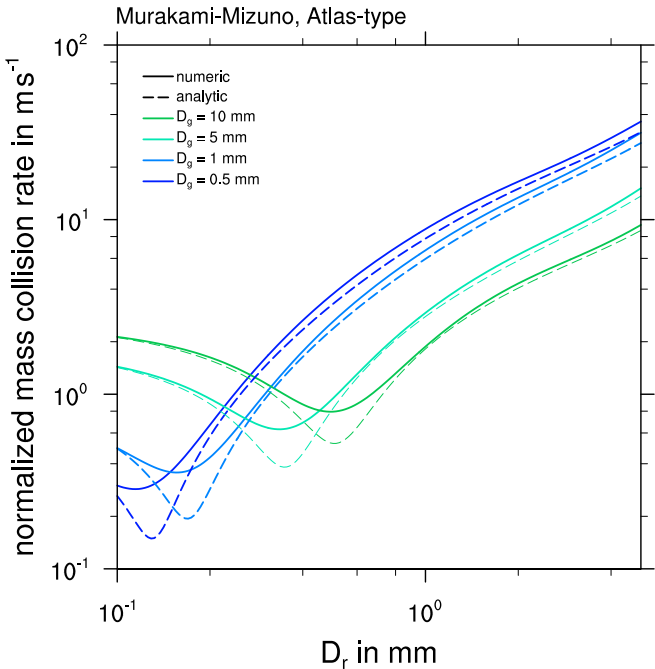

b) SB2006 using power-law fall speed

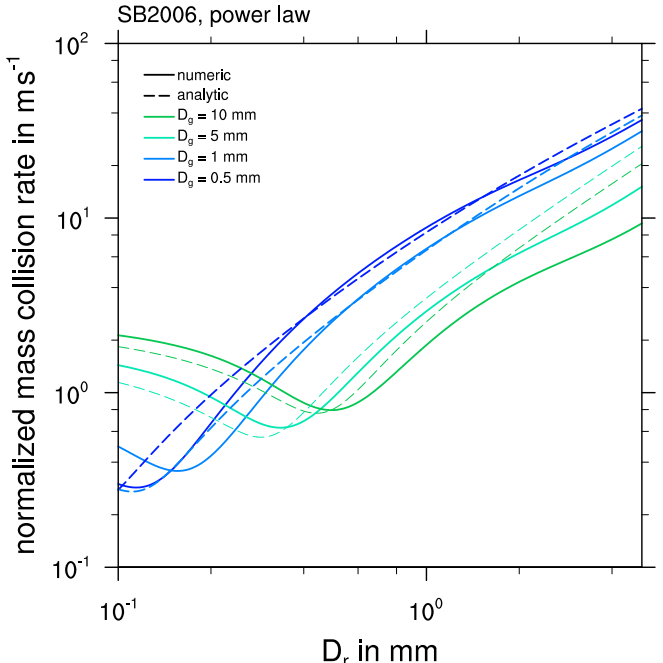

d) Variance approx. using Atlas-type fall speed

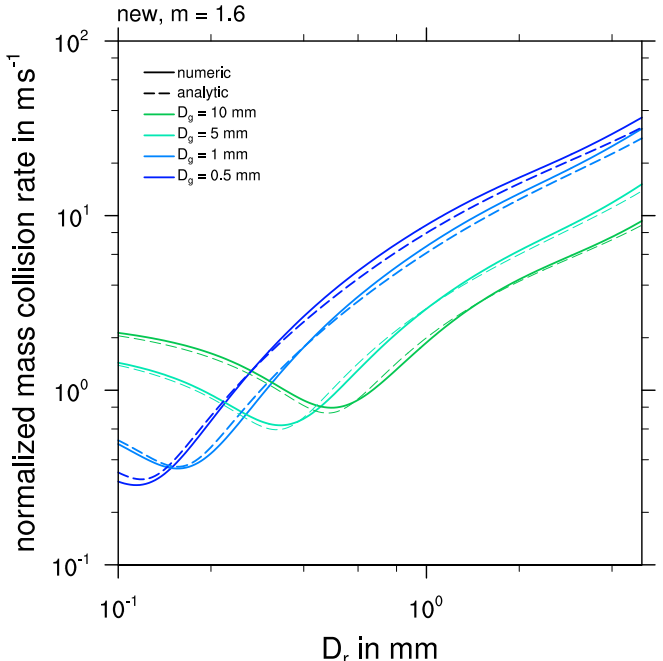

Fig. 4. Normalized mass collision rate for graupel and rain using different approximations (dashed) compared to a numerical solution of the collision integral (solid) as a function of the raindrop mean volume diameter for different mean volume diameters of the graupel size distribution.

$f_{s}\left(D_{\text {eq }}\right)=N_{0, s} D_{\text {eq }}^{\mu_{s}} \exp \left(-\lambda_{s} D_{\text {eq }}\right)$

$x_{s}=a_{s} D_{s}^{2}$

$x_{r}=\frac{\pi}{6} \rho_{w} D_{r}^{3}$

$v_{s}=\alpha_{s}-\beta_{s} \exp \left(-\gamma_{s} D_{\mathrm{eq}}\right)$ $v_{r}=\alpha_{r}-\beta_{r} \exp \left(-\gamma_{r} D_{r}\right)$

$D_{r, \max }=D_{r} \exp \left(\omega_{r} D_{r}\right)$

Note that both $D_{\text {eq }}$ and $D_{r}$ are equivalent diameters, i.e., the diameter of a liquid water sphere. Here $D_{\text {eq }}$ is used for the equivalent diameter of snowflakes and $D_{r}$ for the equivalent diameter of raindrops, while $D_{s}$ and $D_{r, \max }$ are the corresponding maximum dimensions. 
a) Wisner approx. using power-law fall speed

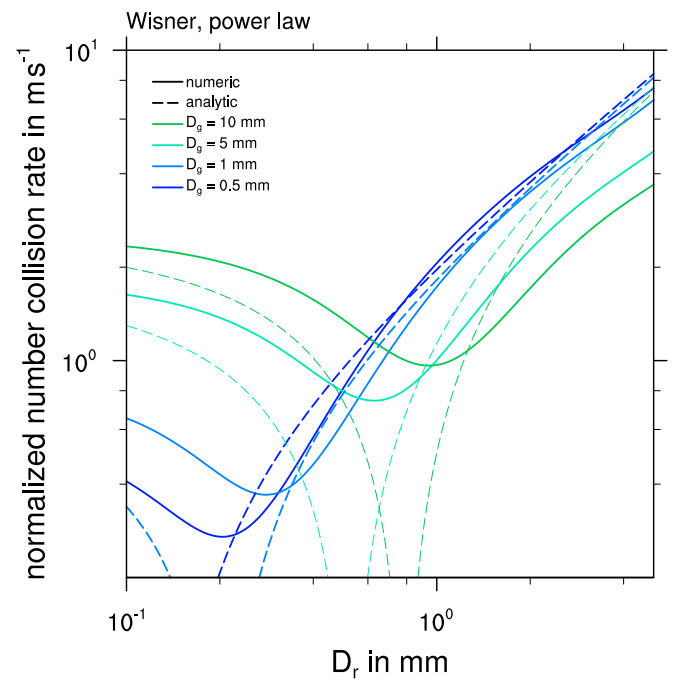

c) Murakami-Mizuno approx. using Atlas-type fall speed

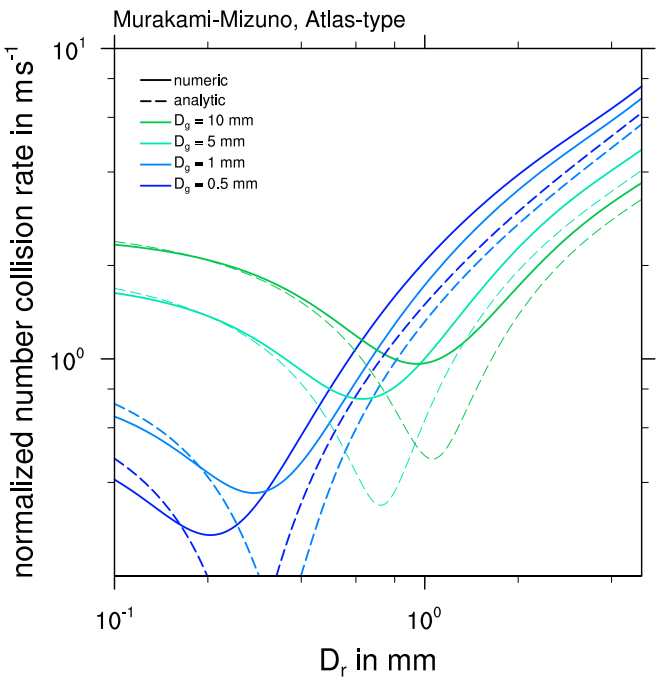

b) SB2006 using power-law fall speed

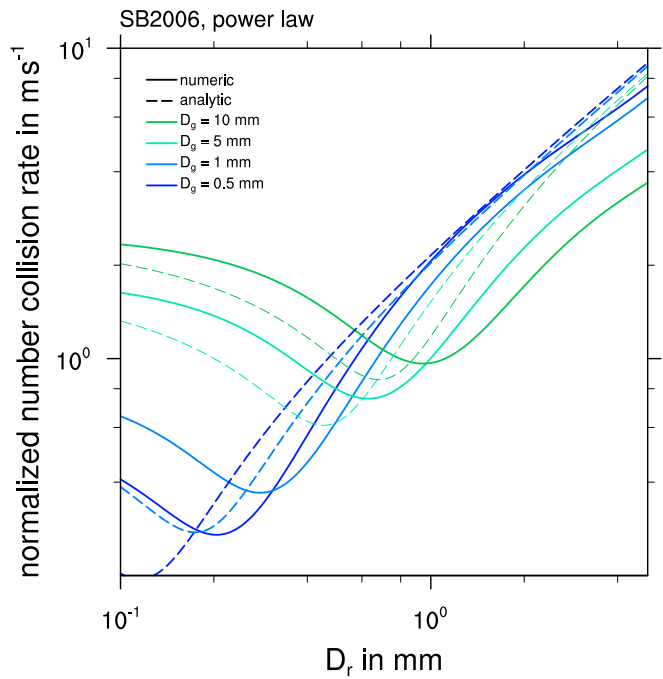

d) Variance approx. using Atlas-type fall speed

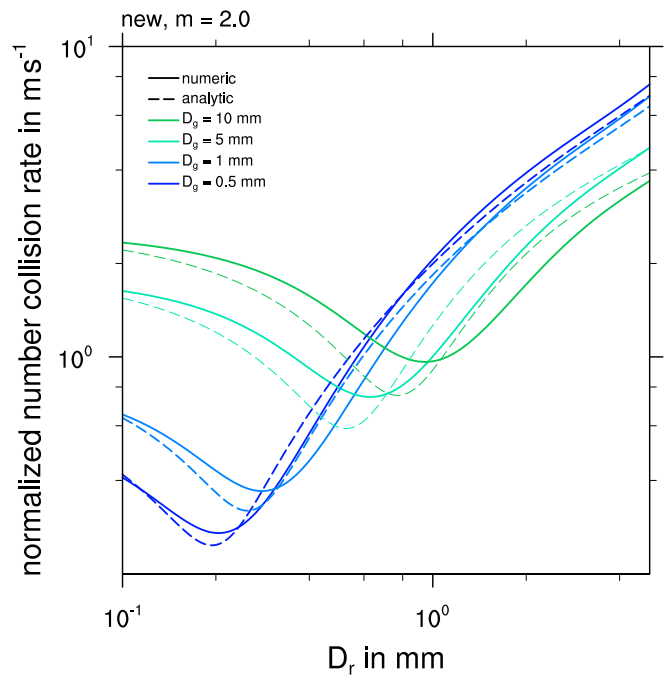

Fig. 5. As Fig. 4 but for the normalized number collision rate of graupel and rain.

For the collection kernel we use the approximation (Connolly et al., 2012)

$$
\begin{aligned}
K\left(D_{r}, D_{\mathrm{eq}}\right) & =\left[A_{r}^{\frac{1}{2}}+A_{s}^{\frac{1}{2}}\right]^{2}\left|v_{r}-v_{s}\right| E_{s r} \\
& =\frac{\pi}{4}\left[D_{r, \max }\left(D_{r}\right)+\hat{a}_{s}^{\frac{1}{2}} D_{s}\left(D_{\mathrm{eq}}\right)\right]^{2} \\
& \times\left|v_{r}\left(D_{r}\right)-v_{s}\left(D_{\mathrm{eq}}\right)\right| E_{s r},
\end{aligned}
$$

which takes into account the area ratio of snow $\hat{a}_{s}$. Alternatively, one could say that for snowflakes $\hat{a}_{s}^{\frac{1}{2}} D_{s}$ is the area-equivalent spherical diameter perpendicular to the fall direction.

With these assumptions we find the bulk collision rates for the number density

$$
\begin{aligned}
& \left.\frac{\partial N_{r}}{\partial t}\right|_{\text {coll }, s r}=-\frac{\pi}{4} N_{s} N_{r} \bar{E}_{s r} \\
& \quad \times\left[\hat{a}_{s} \bar{D}_{s}^{2}+2 \delta_{s}^{*} \bar{D}_{s} \hat{a}_{s}^{\frac{1}{2}}\left(\mu_{r}+1\right) \frac{\lambda_{r}^{\mu+1}}{\left(\lambda_{r}-\omega_{r}\right)^{\mu_{r}+2}}\right. \\
& \left.\quad+\left(\mu_{r}+1\right)\left(\mu_{r}+2\right) \frac{\lambda_{r}^{\mu+1}}{\left(\lambda_{r}-2 \omega_{r}\right)^{\mu_{r}+3}}\right]
\end{aligned}
$$


a) Wisner approx. using power-law fall speeds

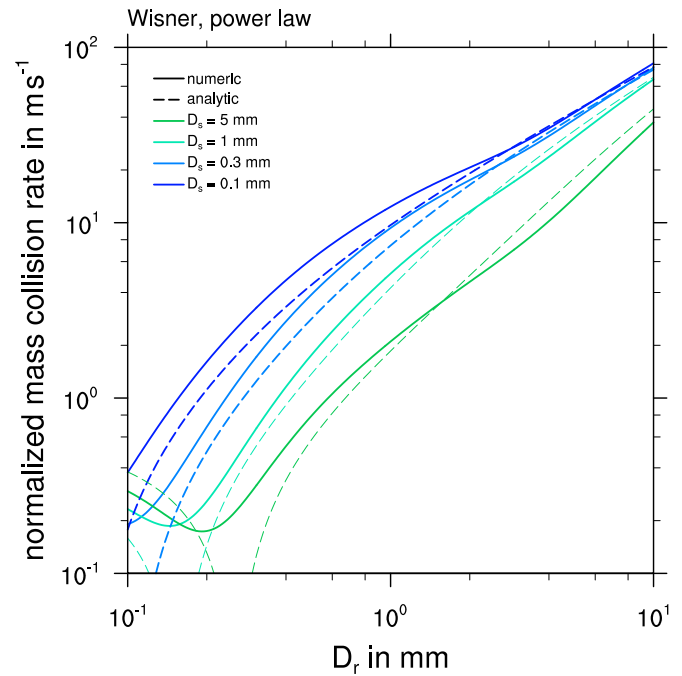

c) Murakami-Mizuno using Atlas-type fall speed

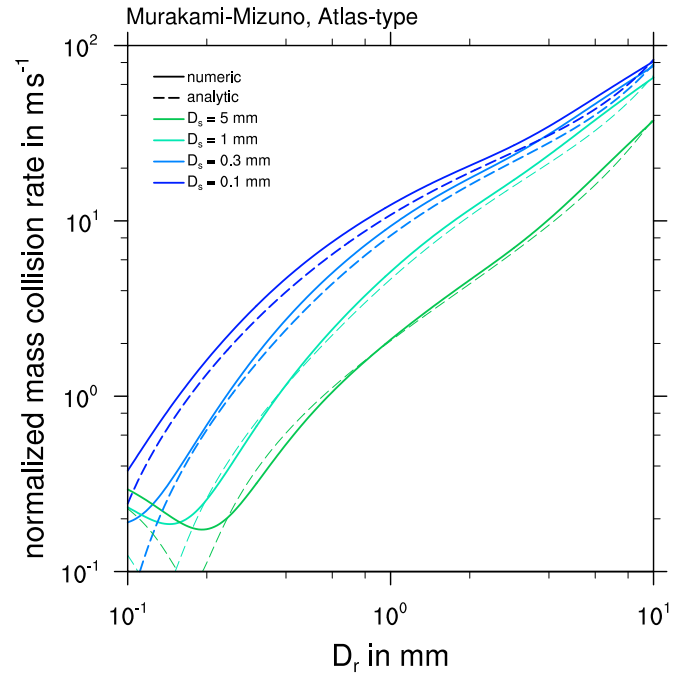

b) SB2006 using power-law fall speeds

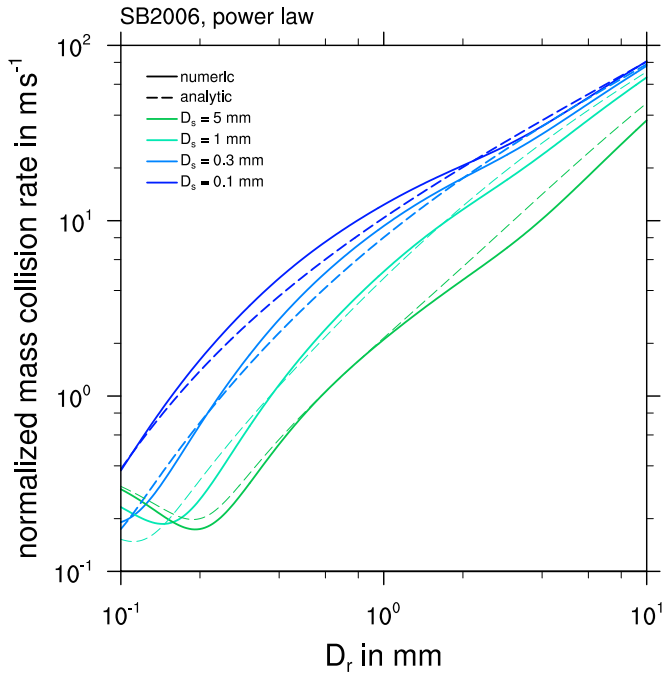

d) Variance approx. using Atlas-type fall speeds

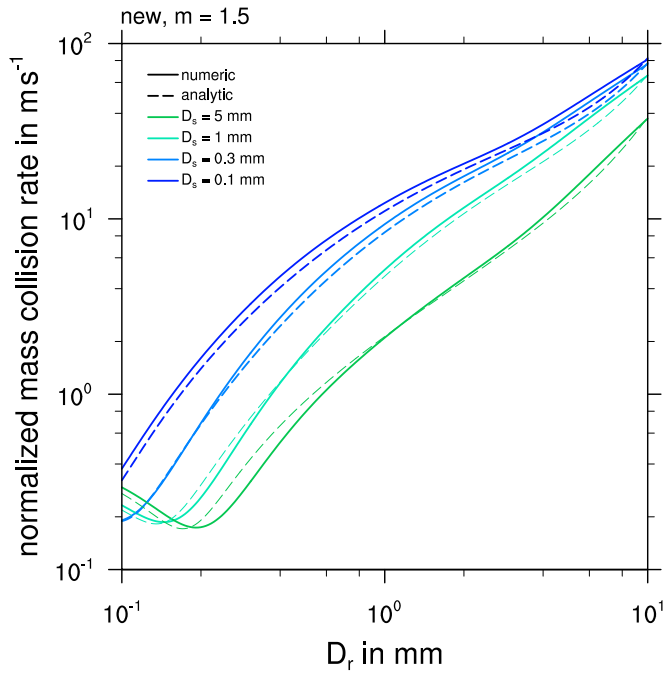

Fig. 6. Normalized mass collision rate for snow and rain using different approximations (dashed) compared to a numerical solution of the collision integral (solid) as a function of the raindrop mean volume diameter for different mean volume diameters of the snow size distribution.

$$
\begin{array}{lrl}
\times\left[\left(\alpha_{s}-\alpha_{r}\right)^{2}-2 \beta_{r}\left(\alpha_{r}-\alpha_{s}\right)\left(1+\frac{\gamma_{r}}{m \lambda_{r}}\right)^{-\left(m \mu_{r}+1\right)}\right. & \text { and mass density } \\
-2 \beta_{s}\left(\alpha_{s}-\alpha_{r}\right)\left(1+\frac{\gamma_{s}}{m \lambda_{s}}\right)^{-\left(m \mu_{s}+1\right)} & \left.\frac{\partial L_{r}}{\partial t}\right|_{\text {coll,sr }}=-\frac{\pi}{4} N_{s} L_{r} \bar{E}_{s r} \\
+\beta_{r}^{2}\left(1+\frac{2 \gamma_{r}}{m \lambda_{r}}\right)^{-\left(m \mu_{r}+1\right)}+\beta_{s}^{2}\left(1+\frac{2 \gamma_{s}}{m \lambda_{s}}\right)^{-\left(m \mu_{s}+1\right)} & \times\left[\hat{a}_{s} \bar{D}_{s}^{2}+2 \delta_{s}^{*} \bar{D}_{s} \hat{a}_{s}^{\frac{1}{2}}\left(\mu_{r}+4\right) \frac{\lambda_{r}^{\mu+4}}{\left(\lambda_{r}-\omega_{r}\right)^{\mu_{r}+5}}\right. \\
\left.-\beta_{r} \beta_{s}\left(1+\frac{\gamma_{r}}{m \lambda_{r}}\right)^{-\left(m \mu_{r}+1\right)}\left(1+\frac{\gamma_{s}}{m \lambda_{s}}\right)^{-\left(m \mu_{s}+1\right)}\right]^{\frac{1}{2}} & \left.+\left(\mu_{r}+4\right)\left(\mu_{r}+5\right) \frac{\lambda_{r}^{\mu+4}}{\left(\lambda_{r}-2 \omega_{r}\right)^{\mu_{r}+6}}\right]
\end{array}
$$


a) Wisner approx. using power-law fall speeds

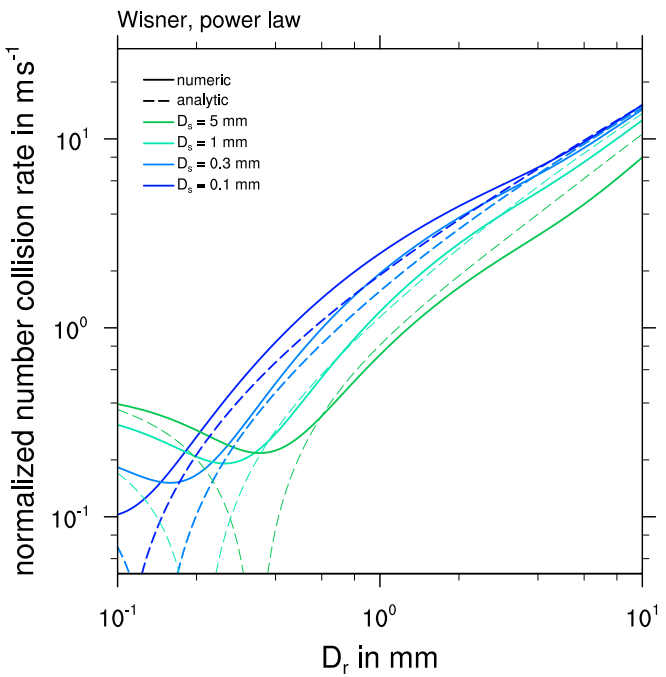

c) Murakami-Mizuno approx. using Atlas-type fall speed

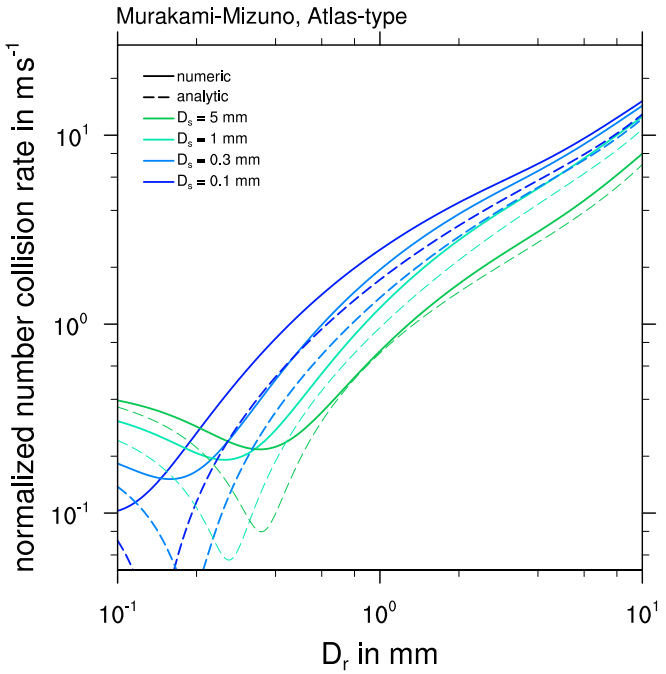

b) SB2006 using power-law fall speeds

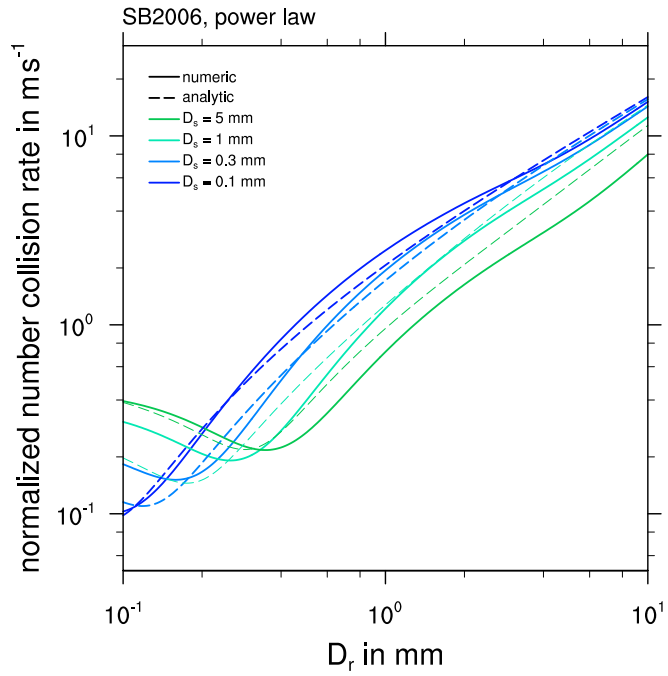

d) Variance approx. using Atlas-type fall speeds

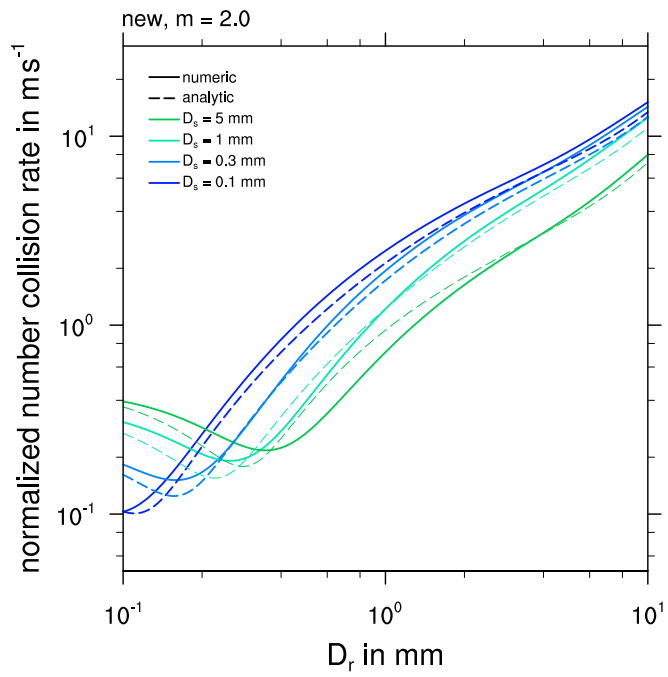

Fig. 7. As Fig. 6 but for the normalized number collision rate of snow and rain.

$$
\begin{aligned}
& \times\left[\left(\alpha_{s}-\alpha_{r}\right)^{2}-2 \beta_{r}\left(\alpha_{r}-\alpha_{s}\right)\left(1+\frac{\gamma_{r}}{m \lambda_{r}}\right)^{-\left(m \mu_{r}+4\right)}\right. \\
& -2 \beta_{s}\left(\alpha_{s}-\alpha_{r}\right)\left(1+\frac{\gamma_{s}}{m \lambda_{s}}\right)^{-\left(m \mu_{s}+1\right)} \\
& +\beta_{r}^{2}\left(1+\frac{2 \gamma_{r}}{m \lambda_{r}}\right)^{-\left(m \mu_{r}+4\right)}+\beta_{s}^{2}\left(1+\frac{2 \gamma_{s}}{m \lambda_{s}}\right)^{-\left(m \mu_{s}+1\right)} \\
& \left.-\beta_{r} \beta_{s}\left(1+\frac{\gamma_{r}}{m \lambda_{r}}\right)^{-\left(m \mu_{r}+4\right)}\left(1+\frac{\gamma_{s}}{m \lambda_{s}}\right)^{-\left(m \mu_{s}+1\right)}\right]^{\frac{1}{2}},
\end{aligned}
$$

with

$$
\delta_{s}^{*}=\frac{\Gamma\left(\mu_{s}+\frac{5}{2}\right)}{\Gamma^{\frac{1}{2}}\left(\mu_{s}+1\right) \Gamma^{\frac{1}{2}}\left(\mu_{s}+4\right)} .
$$

Figures 6 and 7 compare the four different parameterizations for the bulk collision rates. For both rain and snow, we assume a gamma distribution in equivalent diameter with a mass density of $L_{r}=L_{s}=1 \mathrm{~g} \mathrm{~m}^{-3}$, and the shape parameters are $\mu_{r}=2$ and $\mu_{s}=2$. For the collision rate of rain and snow the standard Wisner approximation with power-law fall gives reasonable results except for the minimum of the 
collision rate which occurs for drizzle drops. This is fixed by the SB2006 approximation, which gives a good approximation of the whole size range. The Murakami-Mizuno approximation with Atlas-type fall speeds can improve the collision rates for the very large raindrops when compared to SB2006, but suffers from the underestimation of the minimum. The new variance approximation is therefore the best parameterization as it combines both improvements over the classic Wisner approximation. To achieve the good agreement, the calibration exponent $m$ is necessary; here we find $m=1.5$ for mass and $m=2$ for number.

\section{Self-collection of snow}

Self-collection rates, i.e., loss of particles due to collisions with particles of the same species, cannot be parameterized by the Wisner approximation. Therefore most doublemoment schemes revert to look-up tables and some apply the analytic solution of Passarelli (1978) or the more general one of Verlinde et al. (1990). The analytic solutions are restricted to power-law fall speed relations and include the Gaussian hypergeometric function, which makes them expensive in the case of a time-dependent shape parameter $\mu_{s}$.

With the assumptions

$$
\begin{aligned}
& f_{s}\left(D_{\mathrm{eq}}\right)=N_{0, s} D_{\mathrm{eq}}^{\mu_{s}} \exp \left(-\lambda_{s} D_{\mathrm{eq}}\right) \\
& D_{s} \quad=a_{s}^{-\frac{1}{2}} x_{s}^{\frac{1}{2}}=\left(\frac{\pi \rho_{w}}{6 a_{s}}\right)^{\frac{1}{2}} D_{\mathrm{eq}}^{\frac{3}{2}} \\
& v_{s} \quad=\alpha_{s}-\beta_{s} \exp \left(-\gamma_{s} D_{\mathrm{eq}}\right)
\end{aligned}
$$

the variance approximation can easily be applied to the selfcollection rate of snowflakes, and the resulting parameterization

$$
\begin{aligned}
& \left.\frac{\partial N_{s}}{\partial t}\right|_{\text {coll }, s s}=-\frac{\pi}{2 \sqrt{2}} \beta_{s} N_{s}^{2} \bar{D}_{s}^{2} \hat{a}_{s}\left(1+\delta_{s}^{* 2}\right) \\
& \quad \times\left[\left(1+\frac{2 \gamma_{s}}{m \lambda_{s}}\right)^{-\left(m \mu_{s}+1\right)}-\left(1+\frac{\gamma_{s}}{m \lambda_{s}}\right)^{-2\left(m \mu_{s}+1\right)}\right]^{\frac{1}{2}}
\end{aligned}
$$

is relatively simple and computationally efficient. A comparison with the numerical solution of the integral for $L_{s}=$ $1 \mathrm{~g} \mathrm{~m}^{-3}$ is given in Fig. 8 for three values of the shape parameter $\mu_{s}$. This shows that the parameterization provides a good but not perfect approximation and is able to capture the dependency on the shape parameter $\mu_{s}$ correctly. For the calibration exponent we have chosen $m=1$. Taking into account the Atlas-type fall speed relation is clearly superior to the simple power-law fall speed of the SB2006 formulation which is shown for comparison. The numerical solution using power fall speeds should be very close to the analytic solutions of Passarelli (1978) and Verlinde et al. (1990) and provide also the reference for the SB2006 approximation. This shows that the main advantage of the refined variance approximation comes from the use of the Atlas-type velocity relations.

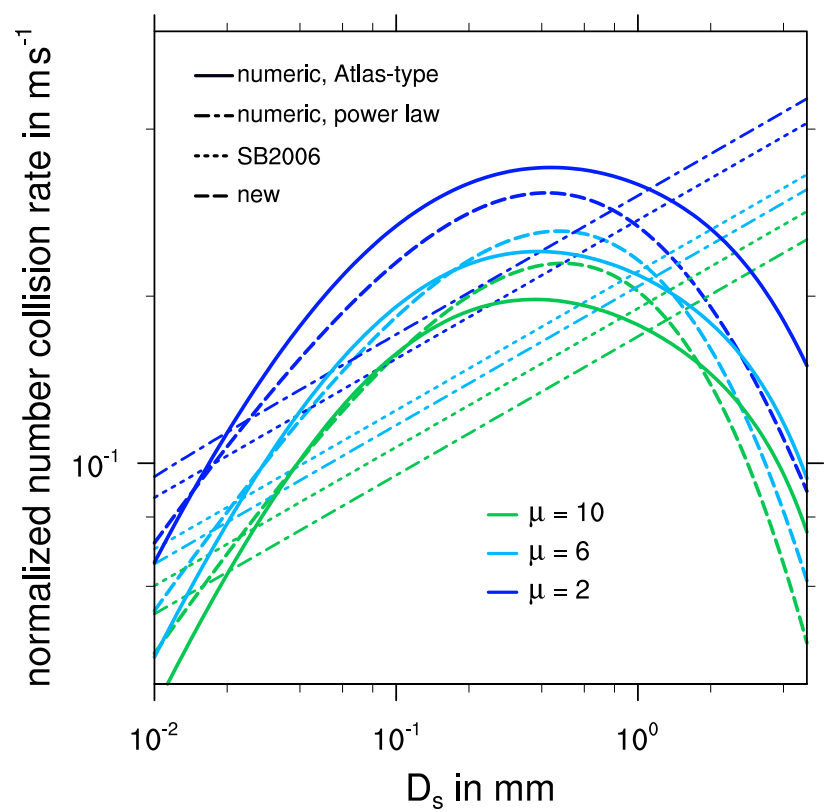

Fig. 8. Normalized bulk number collision rate in $\mathrm{m} \mathrm{s}^{-1}$ for the self-collection of snow as a function of the mean maximum diameter of snowflakes. Shown are the results for three different values of the gamma shape parameter, $\mu_{s}=2, \mu_{s}=6$ and $\mu_{s}=10$, for the SB2006 approximation (dotted), the revised variance approximation using the Atlas-type terminal fall velocity (dashed) and the numerical solution of the integral with Atlas-type (solid) and powerlaw (dash-dotted) fall speed.

\section{Other collection rates}

The previous sections have shown three examples of hydrometeor collision rates which include non-spherical particles, and applying an Atlas-type fall speed approximation and the variance formulation of the differential fall velocity seems to be an appropriate parameterization. This basically applies to all collision rates that include either raindrops or snowflakes. Further examples are discussed in the Supplement. Here we show some quantitative error measures which help to summarize the quality of the various approximations for such collision interactions. As error measures we have chosen the root mean square error (RMSE)

$\operatorname{RMSE}_{\phi, i j}=\sqrt{\frac{1}{\mathfrak{n}} \sum_{\mathfrak{z}=1}^{\mathfrak{n}}\left(\mathcal{K}_{\phi, i j, \text { num }}-\mathcal{K}_{\phi, i j, \text { para }}\right)^{2}}$

and the symmetric mean absolute percentage error (SMAPE)

$\mathrm{SMAPE}_{\phi, i j}=\frac{1}{\mathfrak{n}} \sum_{\mathfrak{z}=1}^{\mathfrak{n}} \frac{\left|\mathcal{K}_{\phi, i j, \text { num }}-\mathcal{K}_{\phi, i j, \text { para }}\right|}{\mathcal{K}_{\phi, i j, \text { num }}+\mathcal{K}_{\phi, i j, \text { para }}}$.

Here $\phi$ can either be $N$ for the number rate or $L$ for the mass rate, and $i, j$ identify the chosen binary collision interaction. Using a simple relative error instead of SMAPE would 
a) RMSE

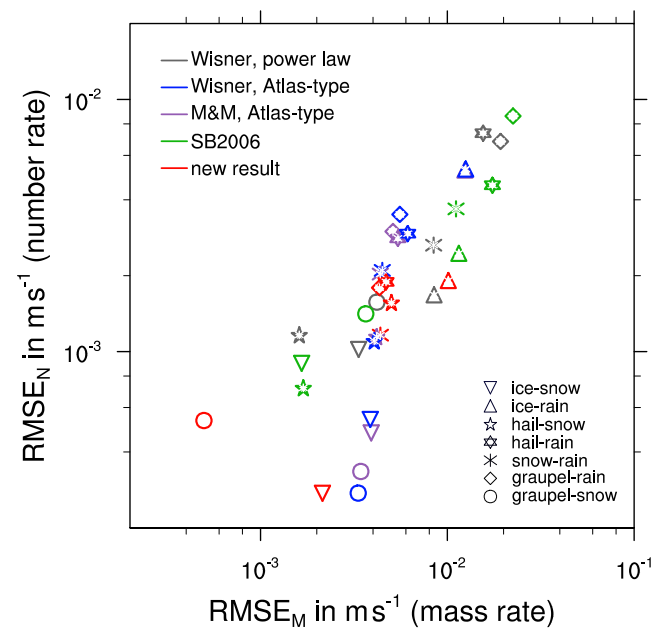

b) SMAPE

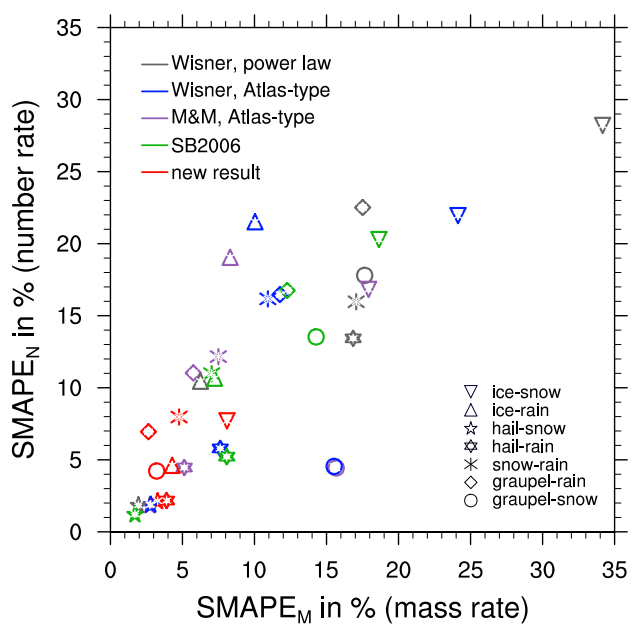

Fig. 9. Root mean square error (RMSE, left) and symmetric mean absolute percentage error (SMAPE, right) of the normalized bulk number and mass collection rates for seven different binary collision interactions.

give an advantage to the Wisner approximation, because in the standard relative error overestimations are more strongly penalized than underestimations. Note that the RMSE depends strongly on the normalization of the rates. Applying the RMSE directly to the number and mass rates can give very misleading results, because then only some asymptotic regime may dominate the error. Using the normalization

$\begin{aligned} \mathcal{K}_{N, i j} & =\left.\frac{1}{N_{i} N_{j}\left(\bar{D}_{i}+\bar{D}_{j}\right)^{2}} \frac{\partial N_{i}}{\partial t}\right|_{\mathrm{coll}, i j} \\ \mathcal{K}_{L, i j} & =\left.\frac{1}{L_{i} N_{j}\left(\bar{D}_{i}+\bar{D}_{j}\right)^{2}} \frac{\partial L_{i}}{\partial t}\right|_{\mathrm{coll}, i j}\end{aligned}$

is an attempt to give the errors of all particle sizes a more similar weight.

The quantitative results for seven different collisions including either rain or snow and other species like graupel, hail or cloud ice in the form of hexagonal plates are shown in Fig. 9. When using SMAPE as a metric, the most difficult collision interaction is the one between cloud ice and snow, because the fall speeds of both species are very similar. The simple Wisner approximation leads to errors in SMAPE of about $20-35 \%$, which can be reduced below $10 \%$ by using the variance parameterization as suggested here. The ansatz of Murakami and Mizuno shows a significant improvement over the Wisner approximation (even for a Wisner approximation which makes use of the Atlas-type fall speed relations). That the variance approximation is slightly worse than the Wisner approximation for the collection of snow by hail is due to the fact that the variance approximation is not asymptotically consistent with the continuous growth solution, at least not perfectly, but for the hail-snow collection the errors of all parameterization are actually small. In that case the Wisner approximation would be, on the one hand, the method of choice if computational efficiency is important. On the other hand, the variance approximation may be more flexible and robust, e.g., in the case of sensitivity studies in which the fall speeds of hail and snow are changed.

\section{Conclusions}

We have presented an approach for the approximation of bulk collision rates of non-spherical hydrometeors undergoing binary collisions. Following Seifert and Beheng (2006) we use the variance approximation of the differential fall speed to arrive at integrals that can be evaluated analytically. The resulting parameterization equations are more complicated than the standard Wisner approximation, which so far has been used in most atmospheric models, but given that those parameterizations are only a very small part of a numerical weather prediction or cloud-resolving model the additional computational expense might be acceptable. The error of the approximations as measured by the symmetric mean absolute percentage error (SMAPE) is in general below 10\%. Given the numerous uncertainties and assumptions in such schemes like particle geometries, terminal fall velocity, collision and sticking efficiencies, particle size distributions, etc., this error seems acceptable.

To achieve the best possible result for a specific collection rate, a calibration of the ansatz using the exponent $m$, (cf., Eq. 20) is necessary, but in most cases $m=1.5$ for mass and $m=2$ for number rates of the interaction of two different species, and $m=1$ for self-collection provides a good approximation. 
Whether the analytic approximations resulting from the variance approach are computationally more efficient than a look-up table will depend on the specific implementation and even more on the detailed architecture, cache size and memory bandwidth of the processor, i.e., whether the performance of the model is already limited by memory bandwidth. In such cases the look-up table leads to additional memory access, which can become a serious performance issue, and the additional floating point operations necessary for the analytic equations of the variance approximation can then be the smaller computational burden.

How the revised and improved formulations of the terminal fall velocities and the bulk collision rates affect the simulations of clouds and precipitation will be investigated in a future study.

The Fortran code to evaluate, test and optimize the variance approximation of the bulk collision integrals for a given set of particle assumptions is available from the corresponding author. It is planned to make this code publicly available as part of the UCLA-LES code at http://gitorious.org/uclales when the new relations have been implemented and tested in the SB two-moment microphysics scheme.

\section{Supplementary material related to this article is available online at http://www.geosci-model-dev.net/7/ 463/2014/gmd-7-463-2014-supplement.pdf.}

Acknowledgements. We thank Klaus D. Beheng for numerous comments on the manuscript and Ann Kristin Naumann for helping us with the implementation of the Nelder-Mead method. We thank Jason Milbrandt and an anonymous reviewer for their comments that helped us to improve the manuscript.

This research was carried out as part of the Hans Ertel Centre for Weather Research. This research network of universities, research institutes and the Deutscher Wetterdienst is funded by the BMVBS (Federal Ministry of Transport, Building and Urban Development).

Edited by: O. Boucher

\section{References}

Abraham, F.: Functional dependence of drag coefficient of a sphere on Reynolds number, Phys. Fluids, 13, 2194-2195, 1970.

Atlas, D., Srivastava, R., and Sekhon, R.: Doppler radar characteristics of precipitation at vertical incidence, Rev. Geophys. Space Phys., 11, 1-35, 1973.

Baldauf, M., Seifert, A., Förstner, J., Majewski, D., and Raschendorfer, M.: Operational convective-scale numerical weather prediction with the COSMO model: description and sensitivities, Mon. Weather Rev., 139, 3887-3905, 2011.

Beard, K. V.: Terminal Velocity and Shape of Cloud and Precipitation Drops Aloft, J. Atmos. Sci., 33, 851-864, 1976.
Beard, K. and Chuang, C.: A new model for the equilibrium shape of raindrops, J. Atmos. Sci., 44, 1509-1524, 1987.

Berry, E. and Reinhardt, R.: Modeling of condensation and collection within clouds, Phys. Sci. Publ., 16, Desert Res. Inst., Univ. of Nevada, 1973.

Best, A.: Empirical formulae for terminal velocity of water drops falling through the atmosphere, Q. J. Roy. Meteorol. Soc., 76, 302-311, 1950 .

Blahak, U.: Accurate approximation of complete and incomplete collision integrals for use in bulk ice phase schemes and application to the riming parameterization, in: Proc. 16th Int. Conf. Clouds and Precip., Leipzig, Germany, 2012.

Böhm, H.: A general equation for the terminal fall speed of solid hydrometeors, J. Atmos. Sci., 46, 2419-2427, 1989.

Böhm, J. P.: A general hydrodynamic theory for mixed-phase microphysics. Part I: drag and fall speed of hydrometeors, Atmos. Res., 27, 253-274, 1992.

Brandes, E. A., Ikeda, K., Zhang, G., Schönhuber, M., and Rasmussen, R. M.: A Statistical and Physical Description of Hydrometeor Distributions in Colorado Snowstorms Using a Video Disdrometer, J. Appl. Meteorol., 46, 634-650, 2007.

Chuang, C. and Beard, K.: A Numerical Model for the Equilibrium Shape of Electrified Raindrops, J. Atmos. Sci., 47, 1374-1389, 1990.

Connolly, P. J., Emersic, C., and Field, P. R.: A laboratory investigation into the aggregation efficiency of small ice crystals, Atmos. Chem. Phys., 12, 2055-2076, doi:10.5194/acp-12-20552012, 2012.

Ćurić, M. and Janc, D.: Analysis of collection equation solutions under truncated size spectrum assumption for hydrometeors, Atmos. Res., 96, 378-387, 2010.

Doms, G. and Schättler, U.: A Description of the Nonhydrostatic Regional Model LM, Part I: Dynamics and Numerics, Offenbach, Germany, 2002.

Field, P., Hogan, R., Brown, P., Illingworth, A., Choularton, T., and Cotton, R.: Parametrization of ice-particle size distributions for mid-latitude stratiform cloud, Q. J. Roy. Meteorol. Soc., 131, 1997-2017, 2005.

Field, P. R., Heymsfield, A. J., and Bansemer, A.: Determination of the Combined Ventilation Factor and Capacitance for Ice Crystal Aggregates from Airborne Observations in a Tropical Anvil Cloud, J. Atmos. Sci., 65, 376-391, 2008.

Frick, C., Seifert, A., and Wernli, H.: A bulk parametrization of melting snowflakes with explicit liquid water fraction for the COSMO model, Geosci. Model Dev., 6, 1925-1939, doi:10.5194/gmd-6-1925-2013, 2013.

Gaudet, B. and Schmidt, J.: Assessment of hydrometeor collection rates from. exact and approximate equations. Part I: A new approximate scheme, J. Atmos. Sci., 62, 143-159, 2005.

Gaudet, B. J. and Schmidt, J. M.: Assessment of hydrometeor collection rates from exact and approximate equations. Part II: Numerical bounding, J. Appl. Meteorol., 46, 82-96, 2007.

Heymsfield, A. J.: Properties of Tropical and Midlatitude Ice Cloud Particle Ensembles. Part I: Median Mass Diameters and Terminal Velocities, J. Atmos. Sci., 60, 2573-2591, 2003.

Kessler, E.: On the distribution and continuity of water substance in atmospheric circulations, Meteor. Monogr. 32, Amer. Meteor Soc., Boston, 1969. 
Khvorostyanov, V. and Curry, J.: Terminal velocities of droplets and crystals: Power laws with continuous parameters over the size spectrum, J. Atmos. Sci., 59, 1872-1884, 2002.

Khvorostyanov, V. and Curry, J.: Fall velocities of hydrometeors in the atmosphere: Refinements to a continuous analytical power law, J. Atmos. Sci., 62, 4343-4357, 2005.

Kogan, Y. and Shapiro, A.: The simulation of a convective cloud in a 3D model with explicite microphysics: Part II - Dynamical and microphysical aspects of cloud merger, J. Atmos. Sci., 53, 2525-2545, 1996.

Lin, Y.-L., Farley, R. D., and Orville, H.: Bulk parameterization of the snow field in a cloud model, J. Clim. Appl. Meteorol., 22, 1065-1092, 1983.

Liu, J. Y. and Orville, H. D.: Numerical modeling of precipitation and cloud shadow effects on mountain induced cumuli, J. Atmos. Sci., 26, 1283-1298, 1969.

Locatelli, J. D. and Hobbs, P. V.: Fall Speeds and Masses of Solid Precipitation Particles, J. Geophys. Res., 79, 2185-2197, 1974.

Mansell, E. R., Ziegler, C. L., and Bruning, E. C.: Simulated Electrification of a Small Thunderstorm with Two-Moment Bulk Microphysics, J. Atmos. Sci., 67, 171-194, 2010.

Milbrandt, J. A. and McTaggart-Cowan, R.: Sedimentation-Induced Errors in Bulk Microphysics Schemes, J. Atmos. Sci., 67, 39313948, 2010.

Milbrandt, J. and Yau, M.: A Multimoment Bulk Microphysics Parameterization. Part I: Analysis of the Role of the Spectral Shape Parameter, J. Atmos. Sci., 62, 3051-3064, 2005a.

Milbrandt, J. and Yau, M.: A Multimoment Bulk Microphysics Parameterization. Part II: A Proposed Three-Moment Closure and Scheme Description, J. Atmos. Sci., 62, 3065-3081, 2005b.

Mitchell, D.: Use of mass- and area-dimensional power laws for determining precipitation particle terminal velocities, J. Atmos. Sci., 53, 1710-1723, 1996.

Mizuno, H.: Parameterization of the Accretion Process between Different Precipitation Elements, J. Meteorol. Soc. Jpn., 68, 395398, 1990

Murakami, M.: Numerical Modeling of Dynamical and Microphysical Evolution of an Isolated Convective Cloud - The 19 July 1981 CCOPE Cloud, J. Meteorol. Soc. Jpn., 68, 107-128, 1990.

Passarelli, R. E.: Theoretical and observational study of snow size spectra and snowflake aggregation efficiencies, J. Atmos. Sci., 35, 882-889, 1978.

Press, W. H., Teukolsky, S. A., Vetterling, W. T., and Flannery, B. P.: Numerical Recipes in FORTRAN, Cambridge University Press, Cambridge, 1992.

Pruppacher, H. R. and Klett, J. D.: Microphysics of Clouds and Precipitation, Kluwer Academic Publishers, Dordrecht, 1997.

Rogers, R. R. and Yau, M. K.: A Short Course in Cloud Physics, Butterworth Heinemann, Woburn, MA, 1996.
Rogers, R. R., Baumgardner, D., Ethier, S. A., Carter, D. A., and Ecklund, W. L.: Comparison of Raindrop Size Distributions Measured by Radar Wind Profiler and by Airplane, J. Appl. Meteorol., 32, 694-699, 1993.

Saleeby, S. M. and Cotton, W. R.: A Binned Approach to CloudDroplet Riming Implemented in a Bulk Microphysics Model, J. Appl. Meteorol. Clim., 47, 694-703, 2008.

Seifert, A.: Parametrisierung wolkenmikrophysikalischer Prozesse und Simulation konvektiver Mischwolken, Ph.D. thesis, Institut für Meteorologie und Klimaforschung, Universität Karlsruhe, Karlsruhe, 2002 (in German).

Seifert, A.: On the parameterization of evaporation of raindrops as simulated by a one-dimensional rainshaft model, J. Atmos. Sci., 65, 3608-3619, 2008

Seifert, A. and Beheng, K.: A two-moment cloud microphysics parameterization for mixed-phase clouds. Part I: Model Description, Meteorol. Atmos. Phys., 92, 45-66, 2006.

Straka, J.: Cloud and precipitation microphysics: principles and parameterizations, Cambridge University Press, 2009.

Straka, J. M. and Gilmore, M. S.: Does the influence of oblatelike distortions in larger raindrops make a difference in collection and evaporation parameterizations?, J. Appl. Meteorol., 45, 1582-1591, 2006.

Szakall, M., Mitra, S. K., Diehl, K., and Borrmann, S.: Shapes and oscillations of falling raindrops - A review, Atmos. Res., 97, 416-425, 2010.

Thompson, G., Field, P. R., Rasmussen, R. M., and Hall, W. D.: Explicit Forecasts of Winter Precipitation Using an Improved Bulk Microphysics Scheme. Part II: Implementation of a New Snow Parameterization, Mon. Weather Rev., 136, 5095-5115, 2008.

Verlinde, J., Flatau, P. J., and Cotton, W. R.: Analytical Solutions to the Collection Growth Equation: Comparison with Approximate Methods and Application to Cloud Microphysics Parameterization Schemes, J. Atmos. Sci., 47, 2871-2880, 1990.

Walko, R. L., Cotton, W. R., Meyers, M. P., and Harrington, J. Y.: New RAMS cloud microphysics parameterization. Part I: The single-moment scheme, Atmos. Res., 38, 29-62, 1995.

Westbrook, C., Ball, R., Field, P., and Heymsfield, A.: Universality in snowflake aggregation, Geophys. Res. Lett., 31, 2004a.

Westbrook, C., Ball, R., Field, P., and Heymsfield, A.: Theory of growth by differential sedimentation, with application to snowflake formation, Phys. Rev. E, 70, 021403 , doi:10.1103/PhysRevE.70.021403, 2004b.

Wilson, D. R. and Ballard, S. P.: A microphysically based precipitation for the UK Meteorological Office Unified Model, Q. J. Roy. Meteorol. Soc., 125, 1607-1636, 1999.

Wisner, C., Orville, H. D., and Myers, C.: A numerical model of a hail-bearing cloud, J. Atmos. Sci., 29, 1160-1181, 1972. 